

\section{Cover:}

Background: Photograph showing U.S. Geological Survey Hydrologic Technician collecting an equal-width-increment (EWI) water sample on the South Fork Newaukum River, Washington. Photograph by R.W. Sheibley, U.S. Geological Survey, June 2015.

Top inset: Photograph showing U.S. Geological Survey Ecologist scraping a rock using a circular template for the analysis of Algal biomass and community composition, June 2015. Photograph by U.S. Geological Survey.

Bottom inset: Photograph showing juvenile coho salmon being measured during a fish survey for the Pacific Northwest Stream Quality Assessment, June 2015. Photograph by Peter van Metre, U.S. Geological Survey. 


\section{Design and Methods of the Pacific Northwest Stream Quality Assessment (PNSQA), 2015}

By Richard W. Sheibley, Jennifer L. Morace, Celeste A. Journey, Peter C. Van Metre, Amanda H. Bell, Naomi Nakagaki, Daniel T. Button, and Sharon L. Qi

National Water-Quality Assessment Project

Open-File Report 2017-1103

U.S. Department of the Interior

U.S. Geological Survey 


\section{U.S. Department of the Interior \\ RYAN K. ZINKE, Secretary}

\section{U.S. Geological Survey \\ William H. Werkheiser, Acting Director}

U.S. Geological Survey, Reston, Virginia: 2017

For more information on the USGS-the Federal source for science about the Earth, its natural and living resources, natural hazards, and the environment-visit https://www.usgs.gov/ or call 1-888-ASK-USGS (1-888-275-8747).

For an overview of USGS information products, including maps, imagery, and publications, visit https:/store.usgs.gov.

Any use of trade, firm, or product names is for descriptive purposes only and does not imply endorsement by the U.S. Government.

Although this information product, for the most part, is in the public domain, it also may contain copyrighted materials as noted in the text. Permission to reproduce copyrighted items must be secured from the copyright owner.

Suggested citation:

Sheibley, R.W., Morace, J.L., Journey, C.A., Van Metre, P.C., Bell, A.H., Nakagaki, Naomi, Button, D.T., and Qi, S.L., 2017, Design and methods of the Pacific Northwest Stream Quality Assessment (PNSQA), 2015: U.S. Geological Survey Open-File Report 2017-1103, 46 p., https://doi.org/10.3133/ofr20171103. 


\section{Foreword}

Sustaining the quality of the Nation's water resources and the health of our diverse ecosystems depends on the availability of sound water-resources data and information to develop effective, science-based policies. Effective management of water resources also brings more certainty and efficiency to important economic sectors. Taken together, these actions lead to immediate and long-term economic, social, and environmental benefits that make a difference to the lives of the almost 400 million people projected to live in the United States by 2050.

In 1991, Congress established the National Water-Quality Assessment (NAWQA) to address where, when, why, and how the Nation's water quality has changed, or is likely to change in the future, in response to human activities and natural factors. Since then, NAWQA has been a leading source of scientific data and knowledge used by national, regional, State, and local agencies to develop science-based policies and management strategies to improve and protect water resources used for drinking water, recreation, irrigation, energy development, and ecosystem needs (https://water.usgs.gov/nawqa/applications/). Plans for the third decade of NAWQA (2013-23) address priority water-quality issues and science needs identified by NAWQA stakeholders, such as the Advisory Committee on Water Information and the National Research Council, and are designed to meet increasing challenges related to population growth, increasing needs for clean water, and changing land-use and weather patterns.

The NAWQA project's third decade of implementation includes assessments of water quality and biological conditions in streams that are focused on ecologically distinct regions of the country. These Regional Stream Quality Assessments (RSQA) simultaneously characterize watershed and stream-reach water-quality stressors along with in-stream biological conditions, in order to better understand stressor-effects relationships at regional scales. In 2015, the NAWQA project conducted the Pacific Northwest Stream Quality Assessment (PNSQA) to investigate stream quality across the Puget Sound and Willamette River Basins from the Canadian border to southcentral Oregon. The report summarizes the study design and methods used in the PNSQA study where a total of 88 streams were sampled. All NAWQA reports are available online at https://water.usgs.gov/nawqa/bib/.

We hope this publication will provide you with insights and information to meet your water-resource needs and will foster increased citizen awareness and involvement in the protection and restoration of our Nation's waters. The information in this report is intended primarily for those interested or involved in resource management and protection, conservation, regulation, and policymaking at the regional and national levels.

Dr. Donald W. Cline Associate Director for Water

U.S. Geological Survey 
This page left intentionally blank 


\section{Contents}

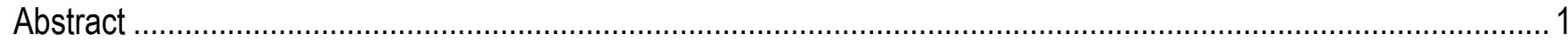

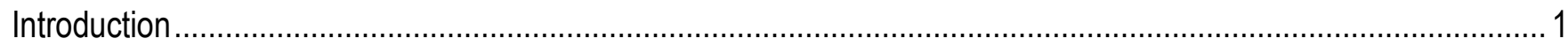

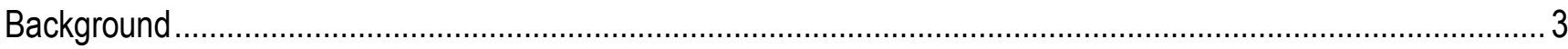

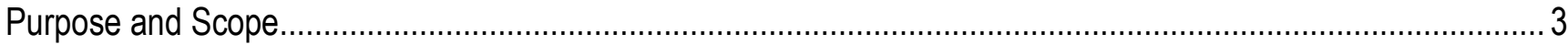

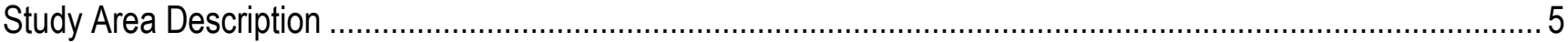

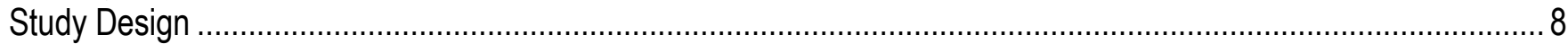

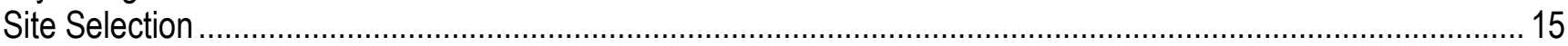

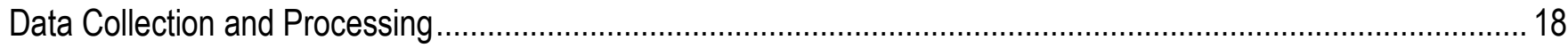

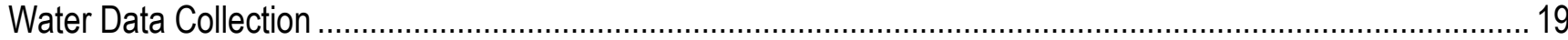

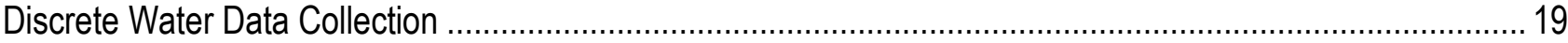

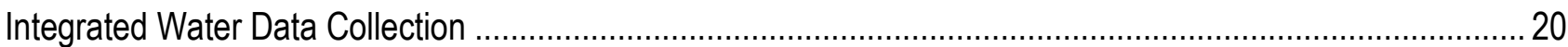

Polar Organic Chemical Integrative Samplers (POCIS) ................................................................. 21

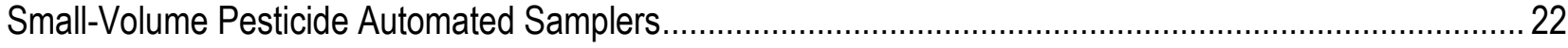

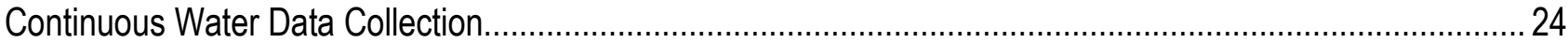

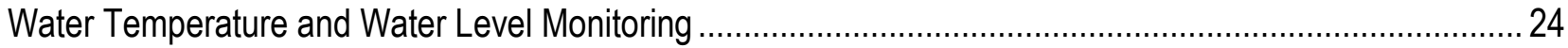

Continuous Water Quality and Algal Biomass ................................................................................... 25

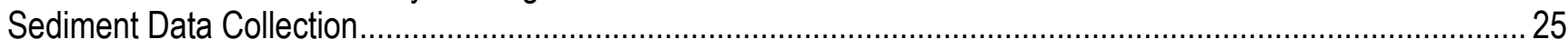

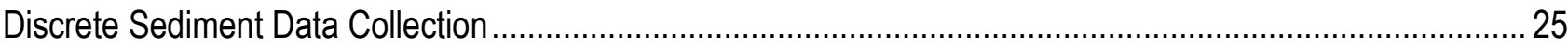

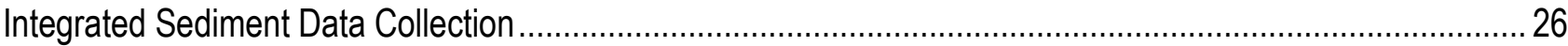

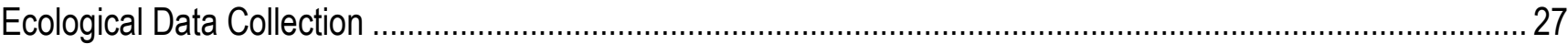

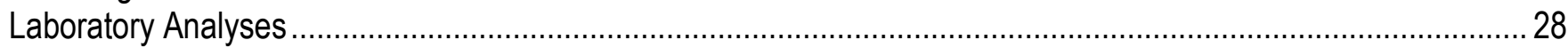

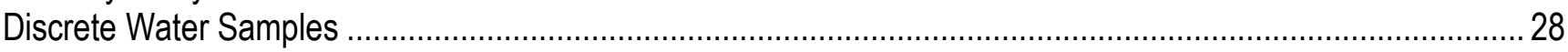

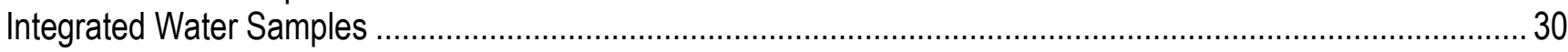

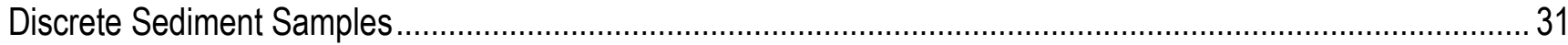

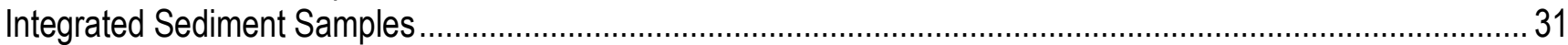

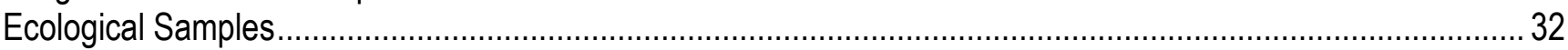

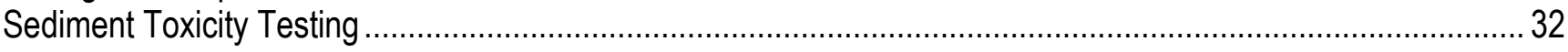

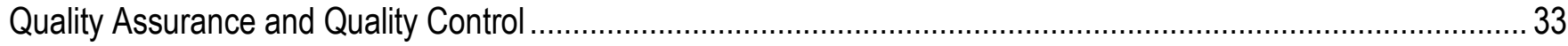

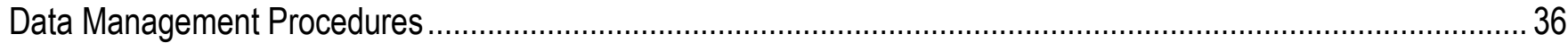

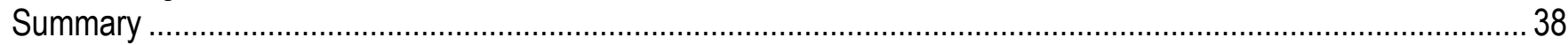

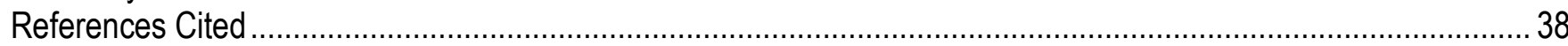

Appendix A. Description of the Sampling Timelines, Matrix, Collection, and Processing for

Water, Sediment, and Ecological Samples

Appendix B. Description of the U.S. Geological Survey Laboratory Schedules Used for Water,

Sediment, and Periphyton 


\section{Figures}

Figure 1. Map showing locations and year of study of the Regional Stream Quality Assessment studies across the United States

Figure 2. Map showing land cover of the Pacific Northwest Stream Quality Assessment study area defined by the Willamette Valley and Puget Lowland Level III Ecoregions in Washington and Oregon, northwestern United States.

Figure 3. Map showing location of agriculture, reference, and urban tier sites sampled for the Pacific Northwest Stream Quality Assessment study, 2015

Figure 4. Ternary diagram showing the distribution of percentage of forested, urban, and agriculture land use in study basins of the Pacific Northwest Stream Quality Assessment.

Figure 5. Flow chart showing sample design for the Pacific Northwest Stream Quality Assessment, 2015

Figure 6. Photographs showing polar organic chemical integrative sampler (POCIS) disks mounted in stainless steel rack and example of protective deployment canister for the field, and deployed POCIS on cinder-block-and-cable infrastructure in Whipple Creek, Washington, 2015

Figure 7. Photographs showing small-volume pesticide automated samplers used to collect filtered water at sub-daily intervals for pesticide analysis as part of the Pacific Northwest Stream Quality Assessment, and examples of field deployments at Bertrand and Thornton Creeks, Washington, 2015.

Figure 8. Photograph showing Walling tubes installed to collect suspended sediment in Rock Creek near Damascus, Oregon

\section{Tables}

Table 1. Average annual precipitation and air temperature at selected stations in the Pacific Northwest Stream Quality Assessment region based on National Oceanic and Atmospheric Administration National Climate Data Center data, 1981-2010

Table 2. Characteristics of Pacific Northwest Stream Quality Assessment sites in the Willamette and Puget Lowland Level III Ecoregions in the northwestern United States, 2015

Table 3. Description of low-, medium-, and high-intensity developed land from datasets in the 2011 National Land Cover Database used to determine urban tiers in the Pacific Northwest Stream Quality Assessment of the U.S. Geological Survey National Water Quality Assessment project, 2015 16

Table 4. Matrix of discrete, integrated, and continuous sampling done at each of the Pacific Northwest Stream Quality Assessment sites, 2015

Table 5. Summary counts of environmental, field blank, replicate, and spike samples of stream water from the 88 stream sites sampled in the Pacific Northwest Stream Quality Assessment study of the U.S. Geological Survey National Water-Quality Assessment Project, 2015 


\section{Conversion Factors}

U.S. customary units to International System of Units

\begin{tabular}{|c|c|c|}
\hline Multiply & By & To obtain \\
\hline \multicolumn{3}{|c|}{ Length } \\
\hline inch (in.) & 2.54 & centimeter $(\mathrm{cm})$ \\
\hline inch (in.) & 25.4 & millimeter $(\mathrm{mm})$ \\
\hline foot (ft) & 0.3048 & meter $(\mathrm{m})$ \\
\hline \multicolumn{3}{|c|}{ Area } \\
\hline square mile $\left(\mathrm{mi}^{2}\right)$ & 259.0 & hectare (ha) \\
\hline square mile $\left(\mathrm{mi}^{2}\right)$ & 2.590 & square kilometer $\left(\mathrm{km}^{2}\right)$ \\
\hline \multicolumn{3}{|c|}{ Volume } \\
\hline gallon (gal) & 3.785 & liter $(\mathrm{L})$ \\
\hline gallon (gal) & 0.003785 & cubic meter $\left(\mathrm{m}^{3}\right)$ \\
\hline \multicolumn{3}{|c|}{ Flow rate } \\
\hline foot per second (ft/s) & 0.3048 & meter per second $(\mathrm{m} / \mathrm{s})$ \\
\hline \multicolumn{3}{|c|}{ International System of Units to U.S. customary units } \\
\hline Multiply & By & To obtain \\
\hline \multicolumn{3}{|c|}{ Length } \\
\hline centimeter $(\mathrm{cm})$ & 0.3937 & inch (in.) \\
\hline millimeter (mm) & 0.03937 & inch (in.) \\
\hline micron $(\mu \mathrm{m})$ & $3.937 \times 10^{-5}$ & inch (in.) \\
\hline nanometer (nm) & $3.937 \times 10^{-8}$ & inch (in.) \\
\hline meter $(\mathrm{m})$ & 3.281 & foot $(\mathrm{ft})$ \\
\hline meter $(\mathrm{m})$ & 1.094 & yard (yd) \\
\hline \multicolumn{3}{|c|}{ Area } \\
\hline square meter $\left(\mathrm{m}^{2}\right)$ & 0.0002471 & acre \\
\hline square meter $\left(\mathrm{m}^{2}\right)$ & 10.76 & square foot $\left(\mathrm{ft}^{2}\right)$ \\
\hline square centimeter $\left(\mathrm{cm}^{2}\right)$ & 0.155 & square inch $\left(\mathrm{ft}^{2}\right)$ \\
\hline \multicolumn{3}{|c|}{ Volume } \\
\hline microliter $(\mu \mathrm{L})$ & $2.462 \times 10^{-7}$ & gallon (gal) \\
\hline milliliter $(\mathrm{mL})$ & 0.0002642 & barrel (petroleum, 1 barrel = 42 gal) \\
\hline liter $(\mathrm{L})$ & 0.2642 & gallon (gal) \\
\hline liter (L) & 61.02 & cubic inch $\left(\mathrm{in}^{3}\right)$ \\
\hline microliter $(\mu \mathrm{L})$ & $6.102 \times 10^{-5}$ & cubic inch $\left(\mathrm{in}^{3}\right)$ \\
\hline \multicolumn{3}{|c|}{ Flow rate } \\
\hline meter per second $(\mathrm{m} / \mathrm{s})$ & 3.281 & foot per second $(\mathrm{ft} / \mathrm{s})$ \\
\hline \multicolumn{3}{|c|}{ Mass } \\
\hline microgram $(\mu \mathrm{g})$ & 1,000 & milligram (mg) \\
\hline milligram (mg) & 0.001 & microgram $(\mu \mathrm{g})$ \\
\hline $\operatorname{gram}(\mathrm{g})$ & 0.03527 & ounce, avoirdupois (oz) \\
\hline kilogram (kg) & 2.205 & pound avoirdupois (lb) \\
\hline
\end{tabular}

Temperature in degrees Celsius $\left({ }^{\circ} \mathrm{C}\right)$ may be converted to degrees Fahrenheit $\left({ }^{\circ} \mathrm{F}\right)$ as ${ }^{\circ} \mathrm{F}=\left(1.8 \times{ }^{\circ} \mathrm{C}\right)+32$.

Temperature in degrees Fahrenheit $\left({ }^{\circ} \mathrm{F}\right)$ may be converted to degrees Celsius $\left({ }^{\circ} \mathrm{C}\right)$ as ${ }^{\circ} \mathrm{C}=\left({ }^{\circ} \mathrm{F}-32\right) / 1.8$. 


\section{Datum}

Horizontal coordinate information is referenced to the North American Datum of 1983 (NAD 83).

\section{Supplemental Information}

Specific conductance is given in microsiemens per centimeter at 25 degrees Celsius $\left(\mu \mathrm{S} / \mathrm{cm}\right.$ at $\left.25^{\circ} \mathrm{C}\right)$.

Concentrations of chemical constituents in water are given either in milligrams per liter $(\mathrm{mg} / \mathrm{L})$, micrograms per liter $(\mu \mathrm{g} / \mathrm{L})$, or nanograms per liter (ng/L).

\section{Abbreviations}

\begin{tabular}{|c|c|c|c|}
\hline AFDW & ash-free dry weight & NWIS & National Water Information System \\
\hline ASR & Analytical Service Request & OGRL & Organic Geochemistry Research Laboratory \\
\hline \multirow[t]{2}{*}{ CC GC/MS } & \multirow{2}{*}{$\begin{array}{l}\text { capillary column gas chromatography/mass } \\
\text { spectrometry }\end{array}$} & OPP & Office of Pesticide Programs \\
\hline & & OTU & operational taxonomic units \\
\hline CERC & \multirow{2}{*}{$\begin{array}{l}\text { Columbia Environmental Research Center } \\
\text { direct aqueous injection }\end{array}$} & $\mathrm{PAH}$ & polycyclic aromatic hydrocarbon \\
\hline DAl & & PBDE & polybrominated diphenyl ether \\
\hline $\mathrm{DQI}$ & data quality indicator & PCFF & Personal Computer Field Form \\
\hline ELISA & enzyme-linked immunosorbent assay & PCB & polychlorinated biphenyl \\
\hline & Environmental Protection Agency & POCIS & polar organic chemical integrative samplers \\
\hline EWI & equal-width increment & PNSQA & Pacific Northwest Stream Quality Assessment \\
\hline ESI & electrospray ionization & PVC & polyvinyl chloride \\
\hline FDOM & fluorescent dissolved organic matter & QA/QC & Quality assurance and quality control \\
\hline GC & gas chromatographic column & $Q C$ & quality control \\
\hline GC/ECNIMS & $\begin{array}{l}\text { gas chromatography electron-capture negative } \\
\text { ionization mode }\end{array}$ & QWDX & Water Quality Data Exchange \\
\hline GC/MS & gas chromatography-mass spectrometry & RTH & richest-targeted habitat \\
\hline GIS & geographic information system & $\mathrm{RP}$ & reference point \\
\hline \multirow[t]{2}{*}{ ICP/AES } & \multirow{2}{*}{$\begin{array}{l}\text { inductively coupled plasma/atomic emission } \\
\text { spectroscopy }\end{array}$} & RSQA & Regional Stream Quality Assessment \\
\hline & & SESQA & Southeast Stream Quality Assessment \\
\hline ICP-MS & inductively coupled plasma-mass spectrometry & TP & total phosphorus \\
\hline INSTAAR & Institute of Arctic and Alpine Research & USGS & U.S. Geological Survey \\
\hline LC-MS/MS & $\begin{array}{l}\text { liquid chromatography tandem mass } \\
\text { spectrometry }\end{array}$ & WMRL & Wisconsin Mercury Research Laboratory \\
\hline LIMS & Laboratory Information Management System & WSC & Water Science Center \\
\hline MDL & method detection limit & & \\
\hline MS & mass spectrometer & & \\
\hline MSQA & Midwest Stream Quality Assessment & & \\
\hline NAWQA & National Water-Quality Assessment & & \\
\hline NHDPlus & National Hydrography Version 2 Dataset & & \\
\hline NLCD & National Land Cover Database & & \\
\hline NWQL & National Water Quality Laboratory & & \\
\hline
\end{tabular}




\title{
Design and Methods of the Pacific Northwest Stream Quality Assessment (PNSQA), 2015
}

\author{
By Richard W. Sheibley, Jennifer L. Morace, Celeste A. Journey, Peter C. Van Metre, Amanda H. Bell,
} Naomi Nakagaki, Daniel T. Button, and Sharon L. Qi

\section{Abstract}

In 2015, the U.S. Geological Survey (USGS) National Water-Quality Assessment (NAWQA) project conducted the Pacific Northwest Stream Quality Assessment (PNSQA) to investigate stream quality across the western part of the Pacific Northwest. The goal of the PNSQA was to assess the health of streams in the region by characterizing multiple water-quality factors that are stressors to instream aquatic life and by evaluating the relation between these stressors and the condition of biological communities. The effects of urbanization and agriculture on stream quality for the Puget Lowland and Willamette Valley Level III Ecoregions were the focus of this regional study. Findings will help inform the public and policymakers about human and environmental factors that are the most critical in affecting stream quality and, thus, provide insights into possible strategies to protect or improve the health of streams in the region.

Land-use data were used in the study to identify and select sites within the region that ranged in levels of urban and agricultural development. A total of 88 sites were selected across the region-69 were on streams that explicitly spanned a range of urban land use in their watersheds, 8 were on streams in agricultural watersheds, and 11 were reference sites with little or no development in their watersheds. Depending on the type of land use, sites were sampled for contaminants, nutrients, and sediment for either a 4- or 10-week period during April, May, and June 2015. This water-quality "index period" was immediately followed with an ecological survey of all sites that included stream habitat, benthic algae, benthic macroinvertebrates, and fish. Additionally, streambed sediment was collected during the ecological survey for analysis of sediment chemistry and toxicity testing.

This report provides a detailed description of the specific study components and methods of the PNSQA, including (1) surveys of stream habitat and aquatic biota, (2) discrete water sampling, (3) deployment of passive polar organic chemical integrative samplers for pesticides and pharmaceuticals, and (4) sampling of streambed sediment. At selected study sites, toxicity testing of streambed sediment, continuous water-quality monitoring, and daily pesticide sampling also were conducted and are described.

\section{Introduction}

The third decade of implementation (Cycle 3; 2013-2023) of the U.S. Geological Survey (USGS) National Water-Quality Assessment (NAWQA) project includes assessments of water quality in streams that are focused on ecologically distinct regions of the country. The Regional Stream Quality Assessment (RSQA) simultaneously characterizes watershed and stream-reach water-quality stressors along with in-stream biological conditions, in order to better understand stressor-effects relationships at regional scales (https://txpub.usgs.gov/RSQA). Each RSQA study is a short-term, multi-stressor 
assessment of stream systems within a targeted, multi-State region (fig. 1). Sampling at RSQA sites spans 4 to 14 weeks during the spring and early summer growing season in order to capture the typical spring pesticide and fertilizer application season.

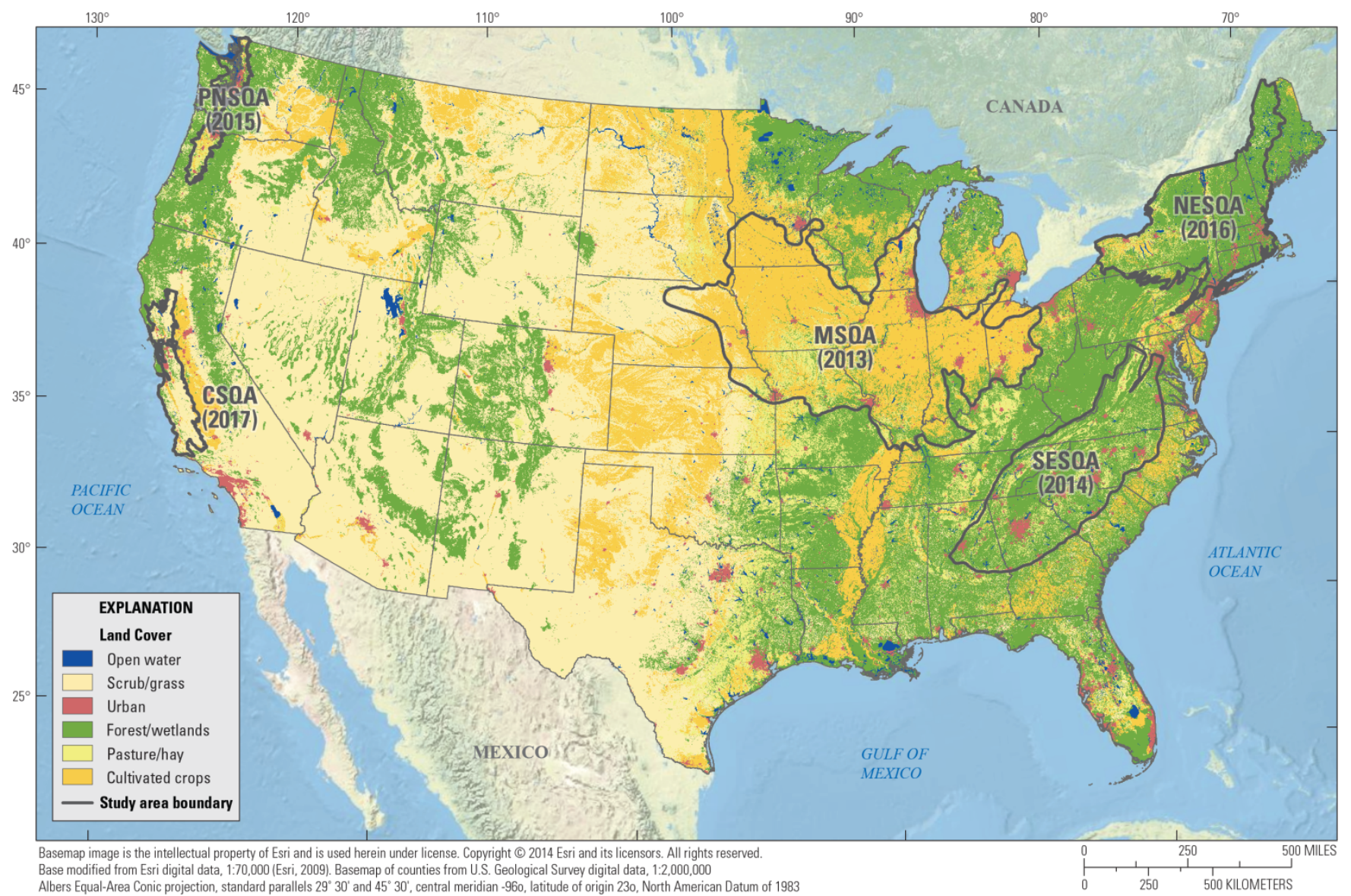

Figure 1. Map showing locations and year of study of the Regional Stream Quality Assessment studies across the United States. MSQA, Midwest Stream Quality Assessment; SESQA, Southeast Stream Quality Assessment; PNSQA, Pacific Northwest Stream Quality Assessment; NESQA, Northeast Stream Quality Assessment; CSQA, California Stream Quality Assessment.

Variations in streamflow, temperature, and sediment and nutrient loads are essential characteristics of natural stream ecosystems, but deviation from their natural patterns can substantially alter biological condition and ecological function (Lenat and Crawford, 1994; Gregory and Calhoun, 2006; Glover and others, 2008; Nagy and others, 2011). Contaminants differ from other stressors in that most are derived from human activities and, through various toxic effects and other modes of action, are potentially detrimental to aquatic life. In order to efficiently manage water resources, it is important to understand the conditions under which individual stressors or combinations of stressors adversely affect stream biological condition and beneficial use designation of water resources for people.

Multi-stressor effects often are assessed in the laboratory under controlled conditions or in the field at small-catchment scales. At these small scales, biogeochemical processes and complex environmental interactions can be manipulated and monitored; however, results of such studies are not readily extended over larger spatial scales. Conversely, biological condition and individual stressors can be evaluated on a national scale (U.S. Environmental Protection Agency, 2006; Herlihy and others, 
2008), and empirical models have been developed to predict metrics of biological condition and environmental stressors across national-scale disturbance gradients (Waite and others, 2000; Klemm and others, 2003; Herlihy and others, 2006; Coles and others, 2012). To date (2016), however, most regional- and national-scale studies have not included a thorough characterization of stressors, but have limited their evaluations to stressor-effect relations. The RSQA studies are designed to bridge this gap by including extensive stressor characterizations at a large number of stream sites and large spatial scales so that development of empirical models can be effectively supported. As such, the studies are intended to provide communities and policymakers with information about the human and environmental factors that have the greatest impact on stream quality.

\section{Background}

In 2015, the NAWQA project assessed stream quality across the western part of the Pacific Northwest region of the United States. The Pacific Northwest Stream Quality Assessment (PNSQA) was the third of the NAWQA Cycle 3 regional studies; the first two studies were the Midwest Stream Quality Assessment (MSQA) in 2013 and the Southeast Stream Quality Assessment (SESQA) in 2014 (Garrett and others, 2017; Journey and others, 2015). The PNSQA study area encompassed watersheds in the Willamette Valley and Puget Lowland Level III Ecoregions (Omernik, 1987, 1995; McMahon and others, 2001) in the States of Oregon and Washington (fig. 2). The PNSQA targeted urbanization in the region because of its rapid population growth that has resulted in urban expansion, and the welldocumented impacts of urban development on aquatic ecosystems (Brown and others, 2005; National Research Council, 2009).

The listing of various populations of anadromous salmonids in the Pacific Northwest as threatened or endangered under the U.S. Endangered Species Act (National Oceanic and Atmospheric Administration, 2016) has had powerful and influential roles (Waples and others, 2013) in directing research and attention on stream ecology across the Pacific Northwest. Few monitoring programs have the capability and history that the NAWQA project provides, and none have been more comprehensive with regard to pesticides (Gilliom and others, 2006) in documenting water-quality conditions of streams and rivers. The data generated by the NAWQA project describing contaminants in freshwater streams have been central to the review of contaminant impacts on threatened or endangered salmonids (Washington State Department of Agriculture, 2001; National Oceanic and Atmospheric Administration, 2012).

\section{Purpose and Scope}

The major objectives of the PNSQA study were to:

1. Determine the status of stream quality across the region on the basis of contaminants, nutrients, sediments, toxicity of the bed sediments, streamflow, habitat, and biological communities;

2. Evaluate the relative influence of contaminants, nutrients, sediment, toxicity, streamflow, and habitat on biological communities in the streams;

3. Evaluate how the natural and anthropogenic characteristics of the watersheds are related to stressors measured at the stream-reach scale, and how the condition of the biological communities can be explained by these stressors; and

4. Develop statistical models and management tools to predict concentrations of contaminants, nutrients, sediment, and ecological health in wadeable streams throughout the region. 


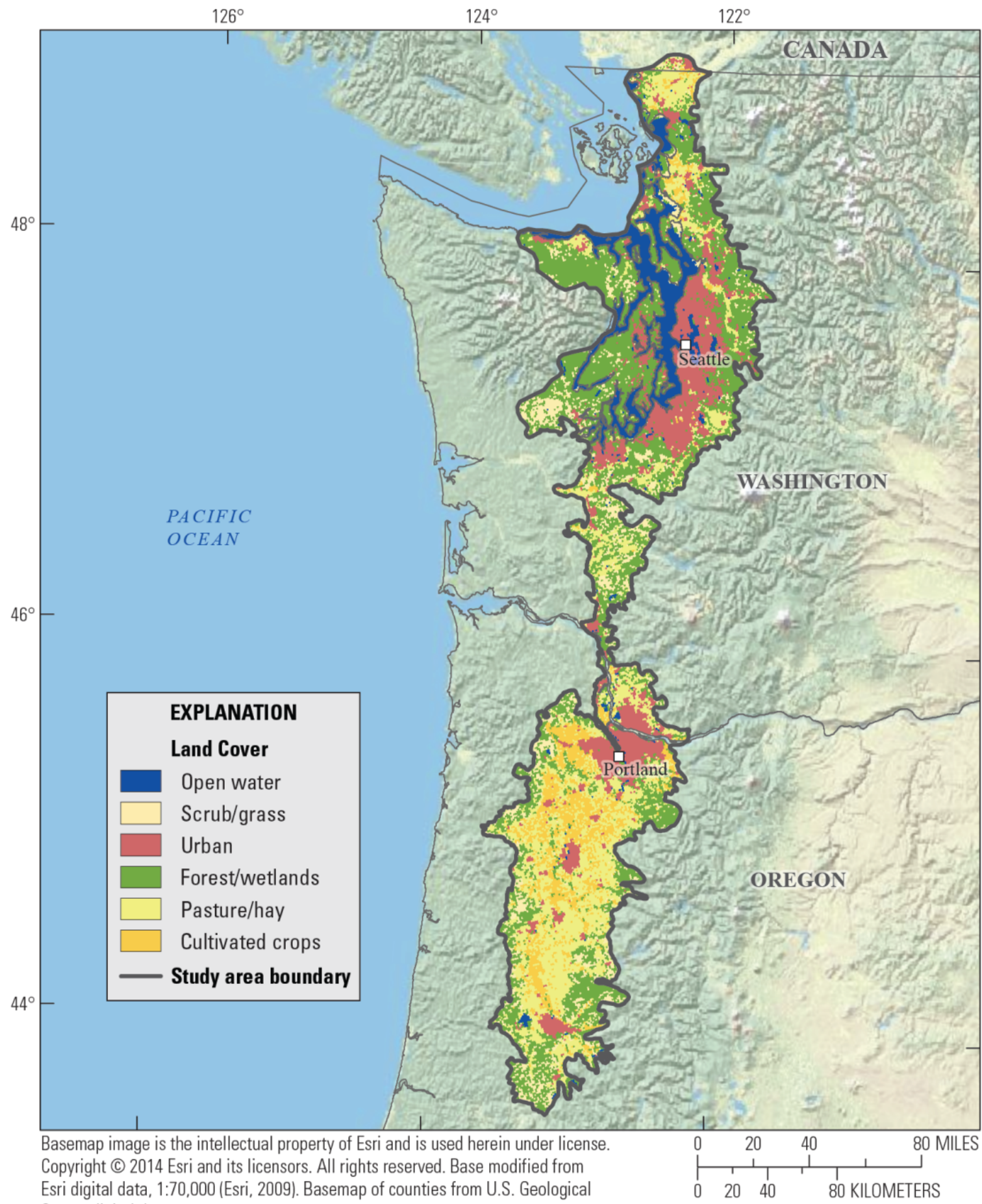

Esri digital data, 1:70,000 (Esri, 2009). Basemap of counties from U.S. Geological

Survey digital data, 1:2,000,000

Albers Equal-Area Conic projection, standard parallels $45^{\circ} 30^{\prime}$ and $60^{\circ} 30^{\prime}$, central

meridian -120 , latitude of origin $23^{\circ}$, North American Datum of 1983

Figure 2. Map showing land cover of the Pacific Northwest Stream Quality Assessment study area defined by the Willamette Valley and Puget Lowland Level III Ecoregions in Washington and Oregon, northwestern United States. 
This report describes the design and methods used in the PNSQA. A network of stream sites was selected throughout the Puget Lowland and Willamette Valley Level III Ecoregions (Omernik, 1987, 1995; McMahon and others, 2001) in the northwestern United States to assess water and sediment quality, biological communities, and habitat integrity during late spring and early summer 2015. Landscape-scale characteristics and in-stream physical and chemical stressors were assessed for their effects on biological communities in the streams.

\section{Study Area Description}

The Puget Lowland Ecoregion covers an area of 6,953 $\mathrm{mi}^{2}$ (Sorenson, 2012) in northwestern Washington State (fig. 2). This ecoregion is bounded by the Coast Range Ecoregion to the west and Cascade Range Ecoregion to the east and follows the Interstate 5 corridor from the Canadian border south to the northern border of the Willamette Valley Ecoregion, near Portland, Oregon. Additionally, the Puget Lowland Ecoregion includes the shoreline of Puget Sound and the San Juan Islands. Elevations within the ecoregion range from sea level to $460 \mathrm{~m}$ and average about $150 \mathrm{~m}$ (DellaSala and others, 2001). Climate in the Puget Lowland Ecoregion is a mild, maritime climate (U.S. Environmental Protection Agency, 1999). Average annual temperatures for 1981-2010 ranged from minimums of 41 to 45 degrees Fahrenheit $\left({ }^{\circ} \mathrm{F}\right)$ to maximums of 56 to $63{ }^{\circ} \mathrm{F}$ (table 1; National Climatic Data Center, 2010). Average annual precipitation in the Puget Lowland- Ecoregion ranged from 19.0 to 65.9 in. during the same period (table 1). Prior to European settlement, the primary land cover in the Puget Lowland Ecoregion was coniferous forests dominated by Douglas-fir (Sorenson, 2012).

Seattle is the largest city in the ecoregion with 608,660 people (U.S. Census Bureau, 2010), and the population of the central Puget Sound region is nearly 4 million people (Puget Sound Regional Council, 2016). From 2015 to 2016, the central Puget Sound had the largest 1-year increase in population this century with an increase of about 87,000 people (Puget Sound Regional Council, 2016). Land-cover change from 1973 to 2000 resulted in a large decrease in forested land cover (about 10 percent) and a large increase in developed land cover (about 7 percent), with agriculture remaining at about the same level (decrease of 0.6 percent) (Sorenson, 2012). In general spatial terms, the center of the Puget Lowland Ecoregion is dominated by developed land cover (Seattle metropolitan area), with agriculture mainly on river floodplains to the north and south, and the remaining outer regions dominated by forests (fig. 2). 
Table 1. Average annual precipitation and air temperature at selected stations in the Pacific Northwest Stream Quality Assessment region based on National Oceanic and Atmospheric Administration National Climate Data Center data, 1981-2010.

[Data compiled from National Climatic Data Center (2010). ${ }^{\circ}$ F, degrees Fahrenheit; Or., Oregon; Wa., Washington]

\begin{tabular}{|c|c|c|c|c|c|c|}
\hline \multirow{2}{*}{ Location of weather station } & \multirow{2}{*}{ State } & \multirow{2}{*}{ Area } & \multirow{2}{*}{$\begin{array}{l}\text { Precipitation } \\
\text { (inches) }\end{array}$} & \multicolumn{3}{|c|}{ Temperature $\left({ }^{\circ} \mathrm{F}\right)$} \\
\hline & & & & Average & High & Low \\
\hline Anacortes & Wa. & Puget Lowland & 27.8 & 52 & 60 & 44 \\
\hline Bellingham Airport & Wa. & Puget Lowland & 35.8 & 50 & 58 & 42 \\
\hline Bremerton & Wa. & Puget Lowland & 56.4 & 52 & 60 & 44 \\
\hline Centralia & Wa. & Puget Lowland & 47.0 & 53 & 62 & 43 \\
\hline Everett & Wa. & Puget Lowland & 38.4 & 52 & 61 & 43 \\
\hline Kent & Wa. & Puget Lowland & 39.1 & 53 & 62 & 45 \\
\hline Longview & Wa. & Puget Lowland & 48.0 & 53 & 62 & 43 \\
\hline Monroe & Wa. & Puget Lowland & 48.8 & 52 & 61 & 43 \\
\hline Mount Vernon & Wa. & Puget Lowland & 33.2 & 51 & 60 & 43 \\
\hline Olympia Airport & Wa. & Puget Lowland & 50.0 & 51 & 60 & 41 \\
\hline Port Townsend & Wa. & Puget Lowland & 19.0 & 52 & 59 & 45 \\
\hline Puyallup & Wa. & Puget Lowland & 40.4 & 53 & 63 & 42 \\
\hline Renton Airport & Wa. & Puget Lowland & 40.1 & 53 & 61 & 45 \\
\hline Seatac Airport & Wa. & Puget Lowland & 37.5 & 53 & 60 & 45 \\
\hline Sedro Woolley & Wa. & Puget Lowland & 46.5 & 51 & 60 & 43 \\
\hline Shelton & Wa. & Puget Lowland & 65.9 & 51 & 61 & 41 \\
\hline Tacoma & Wa. & Puget Lowland & 39.2 & 53 & 62 & 45 \\
\hline Vancouver Airport & Wa. & Puget Lowland & 39.1 & 54 & 63 & 45 \\
\hline Whidbey Island & Wa. & Puget Lowland & 20.3 & 50 & 56 & 43 \\
\hline Median & Wa. & Puget Lowland & 39.2 & 52 & 61 & 43 \\
\hline Minimum & Wa. & Puget Lowland & 19.0 & 50 & 56 & 41 \\
\hline Maximum & Wa. & Puget Lowland & 65.9 & 54 & 63 & 45 \\
\hline Aurora Airport & Or. & Willamette Valley & 41.9 & 54 & 63 & 44 \\
\hline Beaverton & Or. & Willamette Valley & 40.6 & 54 & 64 & 43 \\
\hline Cascadia & Or. & Willamette Valley & 64.5 & 50 & 61 & 40 \\
\hline Corvallis & Or. & Willamette Valley & 42.7 & 53 & 63 & 42 \\
\hline Dallas & Or. & Willamette Valley & 47.0 & 53 & 64 & 42 \\
\hline Eugene Airport & Or. & Willamette Valley & 46.1 & 52 & 63 & 42 \\
\hline Forest Grove & Or. & Willamette Valley & 45.6 & 52 & 63 & 42 \\
\hline Hillsboro & Or. & Willamette Valley & 37.3 & 55 & 65 & 45 \\
\hline Lacomb & Or. & Willamette Valley & 57.2 & 52 & 62 & 41 \\
\hline Lebanon & Or. & Willamette Valley & 46.4 & 54 & 65 & 44 \\
\hline McMinnville Airport & Or. & Willamette Valley & 39.7 & 52 & 63 & 41 \\
\hline Oregon City & Or. & Willamette Valley & 44.2 & 55 & 65 & 45 \\
\hline Portland Airport & Or. & Willamette Valley & 36.0 & 54 & 63 & 46 \\
\hline Salem McNary Field & Or. & Willamette Valley & 39.7 & 53 & 64 & 42 \\
\hline
\end{tabular}




\begin{tabular}{lllcccc}
\hline \multirow{2}{*}{ Location of weather station } & \multirow{2}{*}{ State } & \multicolumn{2}{c}{ Area } & $\begin{array}{c}\text { Precipitation } \\
\text { (inches) }\end{array}$ & \multicolumn{3}{c}{ Temperature $\left({ }^{\circ} \mathrm{F}\right)$} \\
\cline { 6 - 8 } Scappoose Airport & Or. & Willamette Valley & 42.8 & 52 & 63 & 42 \\
Scotts Mills & Or. & Willamette Valley & 80.9 & 49 & 57 & 40 \\
Silver Creek Falls & Or. & Willamette Valley & 71.4 & 48 & 56 & 39 \\
Silverton & Or. & Willamette Valley & 47.4 & 53 & 62 & 44 \\
\hline Median & Or. & Willamette Valley & $\mathbf{4 4 . 9}$ & $\mathbf{5 3}$ & $\mathbf{6 3}$ & $\mathbf{4 2}$ \\
Minimum & Or. & Willamette Valley & $\mathbf{3 6 . 0}$ & $\mathbf{4 8}$ & $\mathbf{5 6}$ & $\mathbf{3 9}$ \\
Maximum & Or. & Willamette Valley & $\mathbf{8 0 . 9}$ & $\mathbf{5 5}$ & $\mathbf{6 5}$ & $\mathbf{4 6}$ \\
\hline & & & & & & \\
\hline Median & All states & All Ecoregions & $\mathbf{4 2 . 7}$ & $\mathbf{5 2}$ & $\mathbf{6 2}$ & $\mathbf{4 3}$ \\
Minimum & All states & All Ecoregions & $\mathbf{1 9 . 0}$ & $\mathbf{4 8}$ & $\mathbf{5 6}$ & $\mathbf{3 9}$ \\
Maximum & All states & All Ecoregions & $\mathbf{8 0 . 9}$ & $\mathbf{5 5}$ & $\mathbf{6 5}$ & $\mathbf{4 6}$ \\
\hline
\end{tabular}

The Willamette Valley Ecoregion covers about 5,582 $\mathrm{mi}^{2}$ (Wilson and Sorenson, 2012). The alluvial Willamette Valley extends north to south between the Coast Range Ecoregion to the west and Cascades Ecoregion to the east (fig. 2). Most of the ecoregion is in Oregon, but the northern extent reaches into southwestern Washington State where the Lewis and Columbia Rivers converge (Wilson and Sorenson, 2012). The Willamette Valley Ecoregion shares its northern border with the Puget Lowland Ecoregion and the southern border with the Klamath Mountains Ecoregion. The topography in the ecoregion is relatively flat, ranging from sea level to $122 \mathrm{~m}$ (Wilson and Sorenson, 2012). Climate in the Willamette Valley Ecoregion is temperate Mediterranean (U.S. Environmental Protection Agency, 1999 ) with average annual temperatures for 1981-2010 ranged from minimums of 39 to $46^{\circ} \mathrm{F}$ to maximums of 56 to $65^{\circ} \mathrm{F}$ (table 1; National Climatic Data Center, 2010). Average annual precipitation in the Willamette Valley Ecoregion ranged from 36.0 in. to 80.9 in. during the same period (table 1).

The flat terrain and fertile soils, mild wet winters, and dry summers, make the Willamette Valley the most important agricultural region in the State (U.S. Environmental Protection Agency, 1999). The largest city in the ecoregion is Portland, with a population of 583,776 in 2010 (U.S. Census Bureau, 2010), and the total population in the Willamette Valley is about 2.3 million (Oregon Department of Fish and Wildlife, 2006). Despite large cities within the valley, the area is a major producer of agriculture and forest products, including grass seed, fruit, nuts, grains, and livestock (Wilson and Sorenson, 2012). Like the Puget Lowland Ecoregion to the north, the greatest decrease in land-cover type in the Willamette Valley Ecoregion between 1973 and 2000 was forest (about 4 percent) and greatest increase in land-cover type was developed land (about 3 percent) (Wilson and Sorenson, 2012). Agricultural land cover decreased by about 2 percent during this same period (Wilson and Sorenson, 2012). 


\section{Study Design}

The PNSQA study incorporated two experimental-design approaches in order to provide different assessments of land-use effects on streams: (1) sites ranked along a land-use gradient, and (2) sites grouped by predominant land uses. The gradient approach was applied to the selection of stream sites that represented a range of urban development across the region, subdivided for design purposes into five urban tiers based on percentages of urban land use. Additionally, a group comparison was used among sites categorized as urban sites, agricultural sites (dominated by agricultural land use), and reference sites (little to no development in their watersheds). These two approaches helped guide the site selection and network design process.

The gradient design used a variation in the land-use intensity approach (for example, Gosz, 1992), a design element often used in NAWQA studies (for example, Coles, and others, 2012) where land use in the watershed is assumed to provide a measure of stressor gradient. Watersheds that lie predominantly or entirely within protected areas (such as parks and wildlife refuges), or are predominately forested (with limited or no urban development), were considered reference conditions. At the other end of the continuum are watersheds in the major urban centers of Seattle and Portland that are subject to high-intensity urban development. For this reason, the impacts of multiple stressors associated with urbanization in the PNSQA study area were assessed by targeting streams that categorized the regional gradient from low- to high-intensity urban development. A total of 80 stream sites were used in the gradient approach, 69 urban tier sites, and 11 reference sites (table 2, fig. 3).

For the group comparison design, agriculture represented an important but comparatively small percentage of the study area, and consequently agricultural land use was a category targeted for comparisons among other land-use groups. Included in the PNSQA were eight sites in agricultural regions of the study area with little urban development (less than 10 percent urban land use) to assess the importance of agricultural land cover as a driver of stream quality. Four of these sites were in Washington and four in Oregon (table 2). The sites in Washington were in the northern extent of the Puget Lowland Ecoregion, primarily in the Skagit and Nooksack river watersheds, and the Oregon sites were spread throughout the Willamette Valley south of Portland (fig 3). The group comparisons included urban tier $(n=69)$ and reference $(n=11)$ sites from the gradient approach with agricultural sites $(\mathrm{n}=8)$, for a total of 88 sites within the Puget Lowland and Willamette Valley Ecoregions (table 2, fig. $3)$. 
Table 2. Characteristics of Pacific Northwest Stream Quality Assessment sites in the Willamette and Puget Lowland Level III Ecoregions in the northwestern United States, 2015.

[Tier 1 sites in bold retain their original classification as Tier 1 sites used during the sample design; however, final watershed data show them having less than 1 percent urban which would be a reference classification. NWIS, USGS National Water Information System database. Latitude and longitude of water quality sample location based on NAD 83 datum and shown in decimal degrees; tier 1-5, urban gradient site with tier 1 with least urbanization and tier 5 with the greatest urbanization; Ag, agriculture; Or., Oregon; Ref, reference; Wa., Washington; --, no data]

\begin{tabular}{|c|c|c|c|c|c|c|c|c|c|c|}
\hline $\begin{array}{l}\text { Map } \\
\text { identi- } \\
\text { fier }\end{array}$ & $\begin{array}{l}\text { NWIS station } \\
\text { number }\end{array}$ & NWIS station name & Field ID & $\begin{array}{l}\text { Urban } \\
\text { center }\end{array}$ & $\begin{array}{l}\text { Site } \\
\text { type }^{1}\end{array}$ & State & Latitude & Longitude & $\begin{array}{c}\text { Drainage } \\
\text { area } \\
\text { (square } \\
\text { miles) }\end{array}$ & $\begin{array}{c}\text { Urban } \\
\text { land use } \\
\text { (percent) }\end{array}$ \\
\hline 1 & 12212001 & $\begin{array}{l}\text { FISHTRAP CREEK AT AARON RD NR } \\
\text { LYNDEN, WA }\end{array}$ & WA_Fishtrap & -- & $\mathrm{Ag}$ & Wa. & 48.96258 & -122.4341 & 21.1 & 1.12 \\
\hline 2 & 12212450 & $\begin{array}{l}\text { BERTRAND CREEK AT WEST } \\
\text { BADGER ROAD NEAR LYNDEN, } \\
\text { WA }\end{array}$ & WA_Bertrand & -- & $\mathrm{Ag}$ & Wa. & 48.96236 & -122.50925 & 28.0 & 1.10 \\
\hline 3 & 12212895 & $\begin{array}{l}\text { TENMILE CREEK ABOVE FOURMILE } \\
\text { CREEK NR FERNDALE, WA }\end{array}$ & WA_Tenmile & -- & $\mathrm{Ag}$ & Wa. & 48.8661 & -122.48171 & 13.6 & 5.82 \\
\hline 4 & 12204010 & $\begin{array}{l}\text { SQUALICUM CREEK NEAR MOUTH } \\
\text { AT BELLINGHAM, WA }\end{array}$ & WA_Squalicum & Bellingham & Tier 3 & Wa. & 48.766 & -122.49967 & 22.3 & 20.10 \\
\hline 5 & 12202300 & $\begin{array}{l}\text { OLSEN CREEK NEAR BELLINGHAM, } \\
\text { WA }\end{array}$ & WA_Olsen & -- & Ref & Wa. & 48.75122 & -122.3535 & 3.9 & 0.43 \\
\hline 6 & 12200017 & $\begin{array}{l}\text { EF NOOKACHAMPS CREEK AT HWY } 9 \\
\text { NR CLEAR LAKE, WA }\end{array}$ & WA_Nooky & -- & $\mathrm{Ag}$ & Wa. & 48.44604 & -122.25238 & 35.5 & 1.66 \\
\hline 7 & 12170000 & $\begin{array}{l}\text { CHURCH CREEK NEAR STANWOOD, } \\
\text { WA }\end{array}$ & WA_Church & Stanwood & Tier 2 & Wa. & 48.2331 & -122.3255 & 11.5 & 13.48 \\
\hline 8 & 12158040 & TULALIP CREEK NEAR TULALIP, WA & WA_Tulalip & Tulalip & Tier 1 & Wa. & 48.06846 & -122.28653 & 15.6 & 8.24 \\
\hline 9 & 12155050 & $\begin{array}{l}\text { DUBUQUE CREEK BLW PANTHER } \\
\text { CREEK NR LK STEVENS, WA }\end{array}$ & WA_Dubuque & Everett & Tier 1 & Wa. & 47.9876 & -122.0343 & 12.8 & 8.42 \\
\hline 10 & 12126910 & $\begin{array}{l}\text { SWAMP CREEK NEAR MOUNTLAKE } \\
\text { TERRACE, WA }\end{array}$ & WA_Swamp & Seattle & Tier 5 & Wa. & 47.79221 & -122.25631 & 20.2 & 72.43 \\
\hline 11 & 12125500 & BEAR CREEK AT WOODINVILLE, WA & WA_BearWood & Redmond & Tier 3 & Wa. & 47.75677 & -122.16513 & 15.5 & 35.51 \\
\hline 12 & 12070000 & $\begin{array}{l}\text { DOGFISH CREEK NEAR POULSBO, } \\
\text { WA }\end{array}$ & WA_Dogfish & Poulsbo & Tier 1 & Wa. & 47.7527 & -122.6437 & 5.9 & 9.76 \\
\hline 13 & 12150495 & $\begin{array}{l}\text { CHERRY CREEK BELOW MARGARET } \\
\text { CREEK NEAR DUVALL, WA }\end{array}$ & WA_Cherry & Duvall & Tier 1 & Wa. & 47.74288 & -121.94123 & 19.4 & 1.90 \\
\hline 14 & 12123100 & $\begin{array}{l}\text { COTTAGE LAKE CREEK AB BEAR } \\
\text { CREEK NEAR REDMOND, WA }\end{array}$ & WA_Cottage & Redmond & Tier 3 & Wa. & 47.71732 & -122.08651 & 11.7 & 29.62 \\
\hline
\end{tabular}




\begin{tabular}{|c|c|c|c|c|c|c|c|c|c|c|}
\hline $\begin{array}{l}\text { Map } \\
\text { identi- } \\
\text { fier }\end{array}$ & $\begin{array}{l}\text { NWIS station } \\
\text { number }\end{array}$ & NWIS station name & Field ID & $\begin{array}{l}\text { Urban } \\
\text { center }\end{array}$ & $\begin{array}{l}\text { Site } \\
\text { type }\end{array}$ & State & Latitude & Longitude & $\begin{array}{l}\text { Drainage } \\
\text { area } \\
\text { (square } \\
\text { miles) }\end{array}$ & $\begin{array}{c}\text { Urban } \\
\text { land use } \\
\text { (percent) }\end{array}$ \\
\hline 15 & 12128040 & $\begin{array}{l}\text { PIPERS CREEK AT CARKEEK PARK, } \\
\text { AT SEATTLE, WA }\end{array}$ & WA_Pipers & Seattle & Tier 5 & Wa. & 47.71149 & -122.37346 & 2.4 & 85.77 \\
\hline 16 & 12120500 & $\begin{array}{l}\text { JUANITA CREEK NEAR KIRKLAND, } \\
\text { WA }\end{array}$ & WA_Juanita & Redmond & Tier 5 & Wa. & 47.70771 & -122.2144 & 6.5 & 77.79 \\
\hline 17 & 12128000 & $\begin{array}{l}\text { THORNTON CREEK NEAR SEATTLE, } \\
\text { WA }\end{array}$ & WA_Thornton & Seattle & Tier 5 & Wa. & 47.69598 & -122.27582 & 12.0 & 86.28 \\
\hline 18 & 12149490 & $\begin{array}{l}\text { HARRIS CREEK ABOVE NE 108TH ST } \\
\text { NR CARNATION, WA }\end{array}$ & WA_Harris & Carnation & Tier 1 & Wa. & 47.69391 & -121.90026 & 7.7 & 8.79 \\
\hline 19 & 12124490 & $\begin{array}{l}\text { BEAR CREEK AT UNION HILL RD AT } \\
\text { REDMOND, WA }\end{array}$ & WA_BearRed & Redmond & Tier 3 & Wa. & 47.67453 & -122.10787 & 46.4 & 27.91 \\
\hline 20 & 12069550 & BEEF CREEK NEAR SEABECK, WA & WA_BigBeef & Seabeck & Tier 1 & Wa. & 47.64065 & -122.78515 & 13.1 & 6.20 \\
\hline 21 & 12120000 & $\begin{array}{l}\text { MERCER CREEK NEAR BELLEVUE, } \\
\text { WA }\end{array}$ & WA_Mercer & Bellevue & Tier 5 & Wa. & 47.60288 & -122.18096 & 12.4 & 71.92 \\
\hline 22 & 12145970 & $\begin{array}{l}\text { PATTERSON CREEK ABV CANYON } \\
\text { CREEK NR FALL CITY, WA }\end{array}$ & WA_Patterson & Fall City & Tier 2 & Wa. & 47.58776 & -121.95327 & 10.6 & 14.83 \\
\hline 23 & 12119705 & COAL CREEK AT BELLEVUE, WA & WA_Coal & Bellevue & Tier 4 & Wa. & 47.56885 & -122.18186 & 6.8 & 44.99 \\
\hline 24 & 12113490 & $\begin{array}{l}\text { LONGFELLOW CREEK AB GENESEE } \\
\text { ST NR WEST SEATTLE, WA }\end{array}$ & WA_Longfellow & Seattle & Tier 5 & Wa. & 47.56453 & -122.36729 & 3.5 & 80.02 \\
\hline 25 & 12121570 & $\begin{array}{l}\text { NORTH FORK ISSAQUAH CREEK AT } \\
\text { ISSAQUAH, WA }\end{array}$ & WA_NFIssaquah & Issaquah & Tier 4 & Wa. & 47.54255 & -122.03461 & 4.7 & 38.20 \\
\hline 26 & 12121504 & $\begin{array}{l}\text { E FORK ISSAQUAH CR ABV 3RD AVE } \\
\text { NE AT ISSAQUAH, WA }\end{array}$ & WA_EFIssaquah & Issaquah & Tier 1 & Wa. & 47.53291 & -122.03088 & 9.3 & 9.02 \\
\hline 27 & 12119495 & $\begin{array}{l}\text { MAY CREEK BELOW HONEY DEW } \\
\text { CREEK NEAR RENTON, WA }\end{array}$ & WA_May & Renton & Tier 3 & Wa. & 47.52066 & -122.19651 & 12.9 & 32.22 \\
\hline 28 & 12068500 & $\begin{array}{l}\text { DEWATTO RIVER NEAR DEWATTO, } \\
\text { WA }\end{array}$ & WA_Dewatto & -- & Ref & Wa. & 47.46898 & -123.0267 & 18.6 & 0.72 \\
\hline 29 & 12120600 & $\begin{array}{l}\text { ISSAQUAH CREEK NEAR HOBART, } \\
\text { WA }\end{array}$ & WA_Issaquah & Hobart & Tier 1 & Wa. & 47.45732 & -122.00512 & 17.9 & 6.31 \\
\hline 30 & 12072660 & $\begin{array}{l}\text { OLALLA CREEK AT BURLEY OLALLA } \\
\text { ROAD NEAR OLALLA, WA }\end{array}$ & WA_Olalla & Olalla & Tier 1 & Wa. & 47.42853 & -122.56828 & 5.0 & 8.58 \\
\hline 31 & 12118525 & $\begin{array}{l}\text { TAYLOR CREEK ABV 236TH AVE SE } \\
\text { AT MAPLE VALLEY, WA }\end{array}$ & WA_Taylor & Kent & Tier 3 & Wa. & 47.4092 & -122.0373 & 4.4 & 23.12 \\
\hline 32 & 12073895 & COULTER CREEK NEAR ALLYN, WA & WA_Coulter & -- & Ref & Wa. & 47.40843 & -122.81709 & 13.0 & 0.73 \\
\hline 33 & 12117695 & $\begin{array}{l}\text { ROCK CREEK AT CEDAR FALLS } \\
\text { ROAD NEAR LANDSBURG, WA }\end{array}$ & WA_RockCedar & -- & Ref & Wa. & 47.40307 & -121.89917 & 3.0 & 0.01 \\
\hline 34 & 12073425 & $\begin{array}{l}\text { MINTER CREEK ABOVE HUGE CREEK } \\
\text { NEAR WAUNA, WA }\end{array}$ & WA_Minter.WQ & Wauna & Tier 2 & Wa. & 47.38956 & -122.69477 & 5.5 & 12.84 \\
\hline
\end{tabular}




\begin{tabular}{|c|c|c|c|c|c|c|c|c|c|c|}
\hline $\begin{array}{c}\text { Map } \\
\text { identi- } \\
\text { fier }\end{array}$ & $\begin{array}{l}\text { NWIS station } \\
\text { number }\end{array}$ & NWIS station name & Field ID & $\begin{array}{l}\text { Urban } \\
\text { center }\end{array}$ & $\begin{array}{l}\text { Site } \\
\text { type }\end{array}$ & State & Latitude & Longitude & $\begin{array}{l}\text { Drainage } \\
\text { area } \\
\text { (square } \\
\text { miles) }\end{array}$ & $\begin{array}{c}\text { Urban } \\
\text { land use } \\
\text { (percent) }\end{array}$ \\
\hline 35 & 12073500 & HUGE CREEK NEAR WAUNA, WA & WA_Huge & Wauna & Tier 1 & Wa. & 47.38914 & -122.69884 & 6.5 & 6.39 \\
\hline 36 & 12113347 & $\begin{array}{l}\text { MILL CREEK AT EARTHWORKS PARK } \\
\text { AT KENT, WA }\end{array}$ & WA_MillKent & Kent & Tier 5 & Wa. & 47.38125 & -122.22058 & 2.6 & 65.55 \\
\hline 37 & 12072679 & $\begin{array}{l}\text { CRESCENT CR AT CRESCENT VAL DR } \\
\text { NR GIG HARBOR, WA }\end{array}$ & WA_Crescent & Gig Harbor & Tier 2 & Wa. & 47.357875 & $\begin{array}{l}- \\
122.578\end{array}$ & 4.9 & 10.43 \\
\hline 38 & 472131122072500 & $\begin{array}{l}\text { LITTLE SOOS CREEK ABV MOUTH NR } \\
\text { MAPLE VALLEY }\end{array}$ & WA_LittleSoos & Kent & Tier 3 & Wa. & 47.35699 & -122.1276 & 7.7 & 20.73 \\
\hline 39 & 12110495 & $\begin{array}{l}\text { JENKINS CREEK NEAR COVINGTION, } \\
\text { WA }\end{array}$ & WA_Jenkins & Kent & Tier 4 & Wa. & 47.3441 & -122.1232 & 17.8 & 42.05 \\
\hline 40 & 12112600 & $\begin{array}{l}\text { BIG SOOS CREEK ABOVE HATCHERY } \\
\text { NEAR AUBURN, WA }\end{array}$ & WA_BigSoos & Kent & Tier 3 & Wa. & 47.31232 & -122.1654 & 71.8 & 34.23 \\
\hline 41 & 12113205 & $\begin{array}{l}\text { MILL CREEK AT PEASLEY CANYON } \\
\text { RD S NR AUBURN, WA }\end{array}$ & WA_MillAuburn & Tacoma & Tier 4 & Wa. & 47.30327 & -122.26489 & 2.7 & 48.45 \\
\hline 42 & 12107950 & $\begin{array}{l}\text { NORTH FORK NEWAUKUM CREEK } \\
\text { NEAR ENUMCLAW, WA }\end{array}$ & $\underset{\text { um }}{\text { WA_NFNewauk }}$ & Enumclaw & Tier 1 & Wa. & 47.23441 & -121.93052 & 1.9 & 0.55 \\
\hline 43 & 12102212 & $\begin{array}{l}\text { SWAN CREEK AT PIONEER WAY } \\
\text { TACOMA, WA }\end{array}$ & WA_Swan & Tacoma & Tier 4 & Wa. & 47.22614 & -122.39291 & 3.6 & 42.26 \\
\hline 44 & 12080800 & $\begin{array}{l}\text { WOODLAND CREEK BELOW } \\
\text { DRAHAM ROAD NEAR LACEY, WA }\end{array}$ & WA_Woodland & Olympia & Tier 5 & Wa. & 47.06354 & -122.8087 & 18.2 & 56.99 \\
\hline 45 & 12027555 & $\begin{array}{l}\text { PRAIRIE CR 0.7 MI UPS FR MOUTH NR } \\
\text { GRAND MOUND, WA }\end{array}$ & WA_Prairie & $\begin{array}{l}\text { Grand } \\
\text { Mound }\end{array}$ & Tier 2 & Wa. & 46.79098 & -123.02247 & 7.6 & 15.63 \\
\hline 46 & 12024000 & $\begin{array}{l}\text { SOUTH FORK NEWAUKUM RIVER } \\
\text { NEAR ONALASKA, WA }\end{array}$ & $\begin{array}{l}\text { WA_SFNewauk } \\
\text { um }\end{array}$ & -- & Ref & Wa. & 46.57566 & -122.68512 & 40.7 & 0.81 \\
\hline 47 & 455122122310600 & $\begin{array}{l}\text { ROCK CREEK NEAR BATTLE } \\
\text { GROUND, WA }\end{array}$ & WA_RockBattle & Vancouver & Tier 1 & Wa. & 45.85133 & -122.52212 & 10.8 & 3.86 \\
\hline 48 & 454904122441800 & $\begin{array}{l}\text { GEE CREEK AT ABRAMS PARK, AT } \\
\text { RIDGEFIELD, WA }\end{array}$ & WA_Gee & Ridgefield & Tier 2 & Wa. & 45.81793 & -122.73872 & 11.9 & 18.29 \\
\hline 49 & 454558122255200 & $\begin{array}{l}\text { SALMON CREEK AT NE 199TH ST, NR } \\
\text { VENERSBORG, WA }\end{array}$ & WA_Salmon & Vancouver & Tier 1 & Wa. & 45.76611 & -122.43124 & 7.5 & 1.65 \\
\hline 50 & 454533122321200 & $\begin{array}{l}\text { WEAVER CR AT SE MERRITT DR, NR } \\
\text { BATTLE GROUND, WA }\end{array}$ & WA_Weaver & Vancouver & Tier 3 & Wa. & 45.75989 & -122.53598 & 6.7 & 27.62 \\
\hline 51 & 454510122424900 & $\begin{array}{l}\text { WHIPPLE CREEK NEAR SALMON } \\
\text { CREEK, WA }\end{array}$ & WA_Whipple & Vancouver & Tier 3 & Wa. & 45.75049 & -122.71677 & 8.6 & 31.91 \\
\hline 52 & 14205400 & $\begin{array}{l}\text { EAST FORK DAIRY CREEK NEAR } \\
\text { MEACHAM CORNER, OR }\end{array}$ & OR_EFDairy & -- & Ref & Or. & 45.68234 & -123.06955 & 33.8 & 0.22 \\
\hline
\end{tabular}




\begin{tabular}{|c|c|c|c|c|c|c|c|c|c|c|}
\hline $\begin{array}{l}\text { Map } \\
\text { identi- } \\
\text { fier }\end{array}$ & $\begin{array}{l}\text { NWIS station } \\
\text { number }\end{array}$ & NWIS station name & Field ID & $\begin{array}{l}\text { Urban } \\
\text { center }\end{array}$ & $\begin{array}{l}\text { Site } \\
\text { type }{ }^{1}\end{array}$ & State & Latitude & Longitude & $\begin{array}{l}\text { Drainage } \\
\text { area } \\
\text { (square } \\
\text { miles) }\end{array}$ & $\begin{array}{c}\text { Urban } \\
\text { land use } \\
\text { (percent) }{ }^{1}\end{array}$ \\
\hline 53 & 14211902 & $\begin{array}{l}\text { BURNT BRIDGE CREEK NEAR } \\
\text { MOUTH AT VANCOUVER, WA }\end{array}$ & WA_Burnt & Vancouver & Tier 5 & Wa. & 45.66164 & -122.66941 & 26.1 & 84.83 \\
\hline 54 & 453506123125700 & $\begin{array}{l}\text { ILER CREEK NEAR FOREST GROVE, } \\
\text { OR }\end{array}$ & OR_Iler & Gales Creek & Tier 1 & Or. & 45.58483 & -123.21706 & 4.9 & 0.18 \\
\hline 55 & 453429122185500 & $\begin{array}{l}\text { GIBBONS CREEK AT EVERGREEN } \\
\text { HWY, AT WASHOUGAL, WA }\end{array}$ & WA_Gibbons & Washougal & Tier 1 & Wa. & 45.57485 & -122.31538 & 7.2 & 8.98 \\
\hline 56 & 453145122534500 & $\begin{array}{l}\text { ROCK CREEK NEAR NW CHERRY } \\
\text { LANE, NEAR ORENCO, OR }\end{array}$ & OR_RockOrenco & Portland & Tier 3 & Or. & 45.52931 & -122.89475 & 25.5 & 25.42 \\
\hline 57 & 453115122540800 & $\begin{array}{l}\text { BEAVERTON CREEK AT NE 75TH } \\
\text { AVE, AT QUATAMA, OR }\end{array}$ & OR_Beaverton & Portland & Tier 5 & Or. & 45.52082 & -122.9021 & 37.2 & 78.07 \\
\hline 58 & 14142800 & BEAVER CREEK AT TROUTDALE, OR & OR_Beaver & Portland & Tier 4 & Or. & 45.5193 & -122.38951 & 10.9 & 44.26 \\
\hline 59 & 452912122291200 & JOHNSON CREEK AT CIRCLE AVE, OR & OR_Johnson & Portland & Tier 3 & Or. & 45.48651 & -122.48787 & 21.1 & 32.64 \\
\hline 60 & 14202920 & SAIN CREEK NEAR GASTON, OR & OR_Sain & -- & Ref & Or. & 45.48039 & -123.24567 & 10.3 & 0.01 \\
\hline 61 & 14211499 & $\begin{array}{l}\text { KELLEY CREEK AT SE 159TH DRIVE } \\
\text { AT PORTLAND, OR }\end{array}$ & OR_Kelley & Portland & Tier 3 & Or. & 45.47701 & -122.49814 & 4.9 & 23.98 \\
\hline 62 & 452543122372300 & $\begin{array}{l}\text { KELLOGG CR BELOW MT SCOTT CR, } \\
\text { NR MILWAUKIE, OR }\end{array}$ & OR_Kellogg & Portland & Tier 5 & Or. & 45.42861 & -122.62314 & 14.7 & 77.02 \\
\hline 63 & 452538122213700 & $\begin{array}{l}\text { NORTH FORK DEEP CR AT SE } \\
\text { CHURCH RD, BORING, OR }\end{array}$ & OR_NFDeep & -- & $\mathrm{Ag}$ & Or. & 45.42729 & -122.3604 & 9.5 & 9.55 \\
\hline 64 & 452431122303200 & $\begin{array}{l}\text { ROCK CREEK NEAR MOUTH, NEAR } \\
\text { DAMASCUS, OR }\end{array}$ & OR_RockMouth & Portland & Tier 3 & Or. & 45.40845 & -122.51009 & 9.2 & 26.58 \\
\hline 65 & 452414122213200 & TICKLE CREEK NEAR BORING, OR & OR_Tickle & Portland & Tier 2 & Or. & 45.40373 & -122.36009 & 12.8 & 11.29 \\
\hline 66 & 14206950 & FANNO CREEK AT DURHAM, OR & OR_Fanno & Portland & Tier 5 & Or. & 45.40345 & -122.75482 & 31.2 & 78.65 \\
\hline 67 & 14194300 & $\begin{array}{l}\text { NORTH YAMHILL RIVER NEAR } \\
\text { FAIRDALE, OR }\end{array}$ & OR_NYamhill & -- & Ref & Or. & 45.36511 & -123.379 & 9.6 & 0.00 \\
\hline 68 & 451818122575500 & $\begin{array}{l}\text { HESS CR AT FOOTPATH, GEORGE } \\
\text { FOX UNIV, NEWBERG, OR }\end{array}$ & OR_Hess & Newberg & Tier 3 & Or. & 45.30525 & -122.96511 & 2.3 & 28.33 \\
\hline 69 & 451350122221100 & $\begin{array}{l}\text { CLEAR CREEK AT METZLER PARK, } \\
\text { NR SPRINGWATER, OR }\end{array}$ & OR_Clear & Springwater & Tier 1 & Or. & 45.23069 & -122.36959 & 32.4 & 0.12 \\
\hline 70 & 451244123050200 & $\begin{array}{l}\text { W.F. PALMER CREEK AT WEBFOOT } \\
\text { ROAD NR DAYTON, OR }\end{array}$ & OR_WFPalmer & -- & $\mathrm{Ag}$ & Or. & 45.21206 & -123.0851 & 7.7 & 4.16 \\
\hline 71 & 451033122275700 & $\begin{array}{l}\text { MILK CREEK AT S DHOOGE RD, } \\
\text { NEAR COLTON, OR }\end{array}$ & OR_Milk & Colton & Tier 1 & Or. & 45.17509 & -122.4657 & 34.9 & 0.34 \\
\hline 72 & 14201300 & $\begin{array}{l}\text { ZOLLNER CREEK NEAR MT ANGEL, } \\
\text { OR }\end{array}$ & OR_Zollner & -- & $\mathrm{Ag}$ & Or. & 45.1004 & -122.82176 & 15.1 & 4.71 \\
\hline
\end{tabular}




\begin{tabular}{|c|c|c|c|c|c|c|c|c|c|c|}
\hline $\begin{array}{c}\text { Map } \\
\text { identi- } \\
\text { fier }\end{array}$ & $\begin{array}{l}\text { NWIS station } \\
\text { number }\end{array}$ & NWIS station name & Field ID & $\begin{array}{l}\text { Urban } \\
\text { center }\end{array}$ & $\begin{array}{l}\text { Site } \\
\text { type }{ }^{1}\end{array}$ & State & Latitude & Longitude & $\begin{array}{l}\text { Drainage } \\
\text { area } \\
\text { (square } \\
\text { miles) }\end{array}$ & $\begin{array}{c}\text { Urban } \\
\text { land use } \\
\text { (percent) }{ }^{1}\end{array}$ \\
\hline 73 & 450033122474700 & $\begin{array}{l}\text { SILVER CR AT SILVER CR } \\
\text { FELLOWSHIP, SILVERTON, OR }\end{array}$ & OR_Silver.WQ & Silverton & Tier 1 & Or. & 45.00907 & -122.79647 & 48.1 & 2.48 \\
\hline 74 & 450016123012800 & $\begin{array}{l}\text { CLAGGETT CREEK AT NORTH RIVER } \\
\text { ROAD AT KEIZER, OR }\end{array}$ & OR_Claggett & Salem & Tier 5 & Or. & 45.00429 & -123.02565 & 9.2 & 92.86 \\
\hline 75 & 445819123042500 & $\begin{array}{l}\text { GIBSON CREEK IN BRUSH COLLEGE } \\
\text { PARK, AT SALEM, OR }\end{array}$ & OR_Brush & Salem & Tier 2 & Or. & 44.97143 & -123.0749 & 5.5 & 10.96 \\
\hline 76 & 445750123032900 & $\begin{array}{l}\text { GLENN CREEK AT HARRITT DR NW, } \\
\text { SALEM, OR }\end{array}$ & OR_Glenn & Salem & Tier 4 & Or. & 44.96379 & -123.05794 & 3.8 & 42.34 \\
\hline 77 & 445551123015800 & PRINGLE CREEK AT SALEM, OR & OR_Pringle & Salem & Tier 5 & Or. & 44.92789 & -123.03281 & 10.5 & 79.69 \\
\hline 78 & 445420123041900 & $\begin{array}{l}\text { CROISAN CREEK AT MADRONA AVE } \\
\text { S, AT SALEM, OR }\end{array}$ & OR_Croisan & Salem & Tier 2 & Or. & 44.90569 & -123.07181 & 4.3 & 13.87 \\
\hline 79 & 445029122592600 & BATTLE CREEK NEAR TURNER, OR & OR_Battle & Salem & Tier 3 & Or. & 44.84123 & -122.99176 & 11.4 & 27.52 \\
\hline 80 & 443808123051100 & $\begin{array}{l}\text { PERIWINKLE CREEK AT SANTIAM RD } \\
\text { SE, AT ALBANY, OR }\end{array}$ & OR_Periwinkle & Albany & Tier 5 & Or. & 44.6359 & -123.08651 & 6.0 & 50.20 \\
\hline 81 & 443436123023600 & $\begin{array}{l}\text { OAK CREEK AT FRY RD SE, NEAR } \\
\text { ALBANY, OR }\end{array}$ & OR_Oak & -- & $\mathrm{Ag}$ & Or. & 44.57655 & -123.0433 & 29.9 & 4.32 \\
\hline 82 & 443423123153700 & $\begin{array}{l}\text { DIXON CREEK AT 6TH ST, AT } \\
\text { CORVALLIS, OR }\end{array}$ & OR_Dixon & Corvallis & Tier 5 & Or. & 44.57311 & -123.26232 & 4.2 & 55.50 \\
\hline 83 & 443243123265400 & $\begin{array}{l}\text { WOODS CREEK AT WOODS CREEK } \\
\text { RD, NEAR PHILOMATH, OR }\end{array}$ & OR_Woods & -- & Ref & Or. & 44.54825 & -123.43263 & 6.4 & 0.19 \\
\hline 84 & 442349122440300 & $\begin{array}{l}\text { AMES CREEK AT HWY 20, AT SWEET } \\
\text { HOME, OR }\end{array}$ & OR_Ames & Sweet Home & Tier 1 & Or. & 44.39718 & -122.73398 & 11.6 & 3.08 \\
\hline 85 & 441205122501000 & $\begin{array}{l}\text { MOHAWK RIVER AT PASCHELKE RD, } \\
\text { NEAR MABEL, OR }\end{array}$ & OR_Mohawk & -- & Ref & Or. & 44.20127 & -122.83607 & 79.3 & 0.02 \\
\hline 86 & 441033122524900 & $\begin{array}{l}\text { PARSONS CREEK AT PARSONS } \\
\text { CREEK RD, NR MARCOLA, OR }\end{array}$ & OR_Parsons & Marcola & Tier 1 & Or. & 44.1758 & -122.88074 & 15.5 & 0.06 \\
\hline 87 & 440656122491200 & $\begin{array}{l}\text { CAMP CREEK AT UPPER CAMP CR } \\
\text { RD, NR WALTERVILLE, OR }\end{array}$ & OR_Camp & -- & Ref & Or. & 44.11677 & -122.8182 & 10.2 & 0.00 \\
\hline 88 & 440257123103200 & $\begin{array}{l}\text { AMAZON CREEK NEAR DANEBO } \\
\text { ROAD AT EUGENE, OR }\end{array}$ & OR_Amazon & Eugene & Tier 5 & Or. & 44.04899 & -123.17842 & 19.4 & 56.25 \\
\hline
\end{tabular}

${ }^{1}$ Urban tier categories defined as ranges of percent urban land use in a watershed computed as the cumulative percentage of low-, medium-, and high-intensity developed land from the 2006 National Land Cover Database (NLCD; classes 22, 23,24): Tier 1, 1-10 percent ; tier 2, greater than (>)10 to 20 percent, tier 3, >20 to 37.5 percent; tier $4,>37.5$ to 50 percent; and tier $5,>50$ percent. 


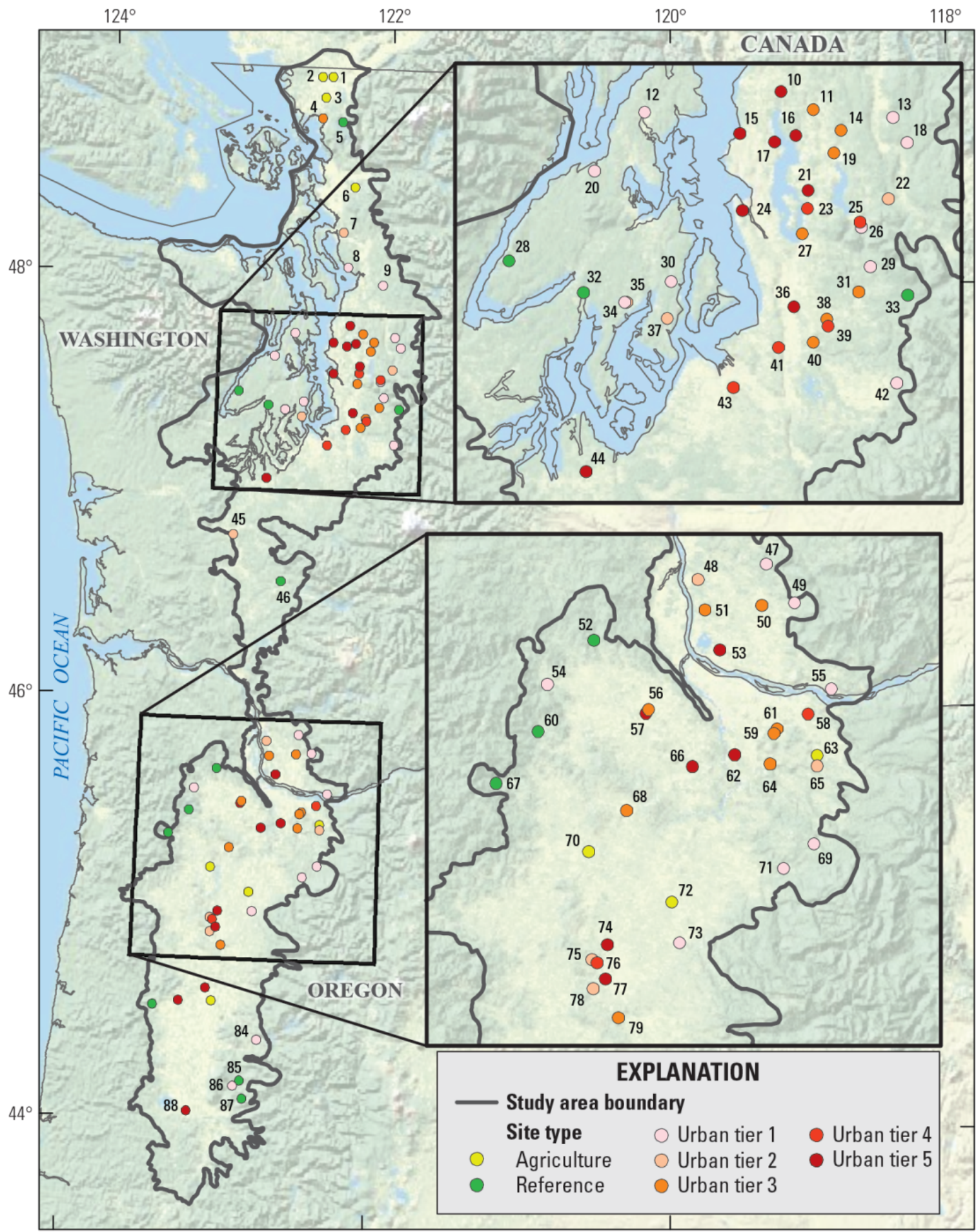

Basemap image is the intellectual property of Esri and is used herein under license. Copyright (C) 2014 Esri and its licensors. All rights reserved. Base modified from Esri digital data, 1:70,000 (Esri, 2009). Basemap of counties from U.S. Geological

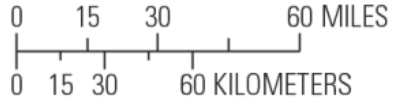

Survey digital data, 1:2,000,000Albers Equal-Area Conic projection, standard parallels $45^{\circ} 30^{\prime}$ and $60^{\circ} 30^{\prime}$, central meridian $-120^{\circ}$, latitude of origin $23^{\circ}$, North American Datum of 1983

Figure 3. Map showing location of agriculture, reference, and urban tier sites sampled for the Pacific Northwest Stream Quality Assessment study, 2015. 


\section{Site Selection}

Perennial, wadeable stream sites were selected for the PNSQA study. A candidate list of sites was first developed on the basis of active and historical (inactive) USGS streamgages, USGS waterquality sampling sites, and streamflow monitoring sites used by State and local agencies. A geospatial database was developed that included land-use and land-cover characteristics for the watersheds of all candidate sites. Watershed delineations and characteristics were available for active USGS streamgages and were determined for other candidate sites using catchment boundaries from the National Hydrography Dataset (NHDPlus Version 2) (U.S. Environmental Protection Agency and U.S. Geological Survey, 2012). Nationally available, digital geographic information system (GIS) data layers (for example, the National Land Cover Database [NLCD]; Homer and others, 2015) were overlain on the delineated watersheds and on the sub-watersheds contributing to each stream segment in NHDPlus for the region. For candidate sites without delineated watersheds, site locations were overlain on the NHDPlus stream network to identify the segment associated with each site. Watershed characteristics for the sites were determined by "accumulating" the area and characteristics of all contributing segment (upstream) sub-watersheds.

Urban, agricultural, and reference sites were selected based on percentages of land use characteristics in their watersheds and with priority given to sites with active streamgages and historical water-quality and ecological data. Candidate urban-gradient stream sites were categorized on the basis of percentage of urban land use calculated as the sum of low-, medium-, and high-intensity developed land in the watershed using the 2011 NLCD data (U.S. Geological Survey, 2014; Homer and others, 2015). Urban sites were distributed among five "tiers" representing the gradient in urban land use:

- Urban tier 1, 1-10 percent;

- Urban tier 2, greater than $(>) 10$ to 20 percent;

- Urban tier 3,>20 to 37.5 percent;

- Urban tier $4,>37.5$ to 50 percent; and

- Urban tier 5, >50 percent (table 3; fig. 4). 
Agricultural sites were selected to represent the major crop types in the region with priority given to settings where agricultural chemical use was expected to be high. A gradient in agricultural land use was not attempted, but, instead, these sites were selected as indicators of some of the more intensive agricultural settings. Reference sites were targeted on the basis of watersheds having a low degree of agricultural ( $<10$ percent) and urban $(<1$ percent) development (table 2$)$ and, wherever possible, the watershed predominantly or entirely within protected lands.

Table 3. Description of low-, medium-, and high-intensity developed land from datasets in the 2011 National Land Cover Database used to determine urban tiers in the Pacific Northwest Stream Quality Assessment of the U.S. Geological Survey National Water Quality Assessment project, 2015.

[More information is available at https:/www.mrlc.gov. NLCD, National Land Cover Database; \%, percent]

\begin{tabular}{|c|c|c|}
\hline $\begin{array}{l}\text { NLCD } \\
\text { classification } \\
\text { code }\end{array}$ & NLCD classification & NLCD classification description \\
\hline 22 & Developed, low intensity & $\begin{array}{l}\text { Areas with a mixture of constructed materials and vegetation. } \\
\text { Impervious surfaces account for } 20-49 \% \text { of the total cover. } \\
\text { These areas most commonly include single-family housing units. }\end{array}$ \\
\hline 23 & Developed, medium intensity & $\begin{array}{l}\text { Areas with a mixture of constructed materials and vegetation. } \\
\text { Impervious surfaces account for } 50-79 \% \text { of the total cover. } \\
\text { These areas most commonly include single-family housing units. }\end{array}$ \\
\hline 24 & Developed, high intensity & $\begin{array}{l}\text { Highly developed areas where people reside or work in high numbers. } \\
\text { Examples include apartment complexes, row houses and } \\
\text { commercial/industrial. Impervious surfaces account for } \\
80-100 \% \text { of the total cover. }\end{array}$ \\
\hline \multicolumn{3}{|c|}{ Definition of urban tiers for PNSQA based on cumulative percentage of classes 22,23 , and 24} \\
\hline & Urban Tier 1 & $1-10 \%$ \\
\hline & Urban Tier 2 & greater than $(>) 10$ to $20 \%$ \\
\hline & Urban Tier 3 & $>20$ to $37.5 \%$ \\
\hline & Urban Tier 4 & $>37.5$ to $50 \%$ \\
\hline & Urban Tier 5 & $>50 \%$ \\
\hline
\end{tabular}




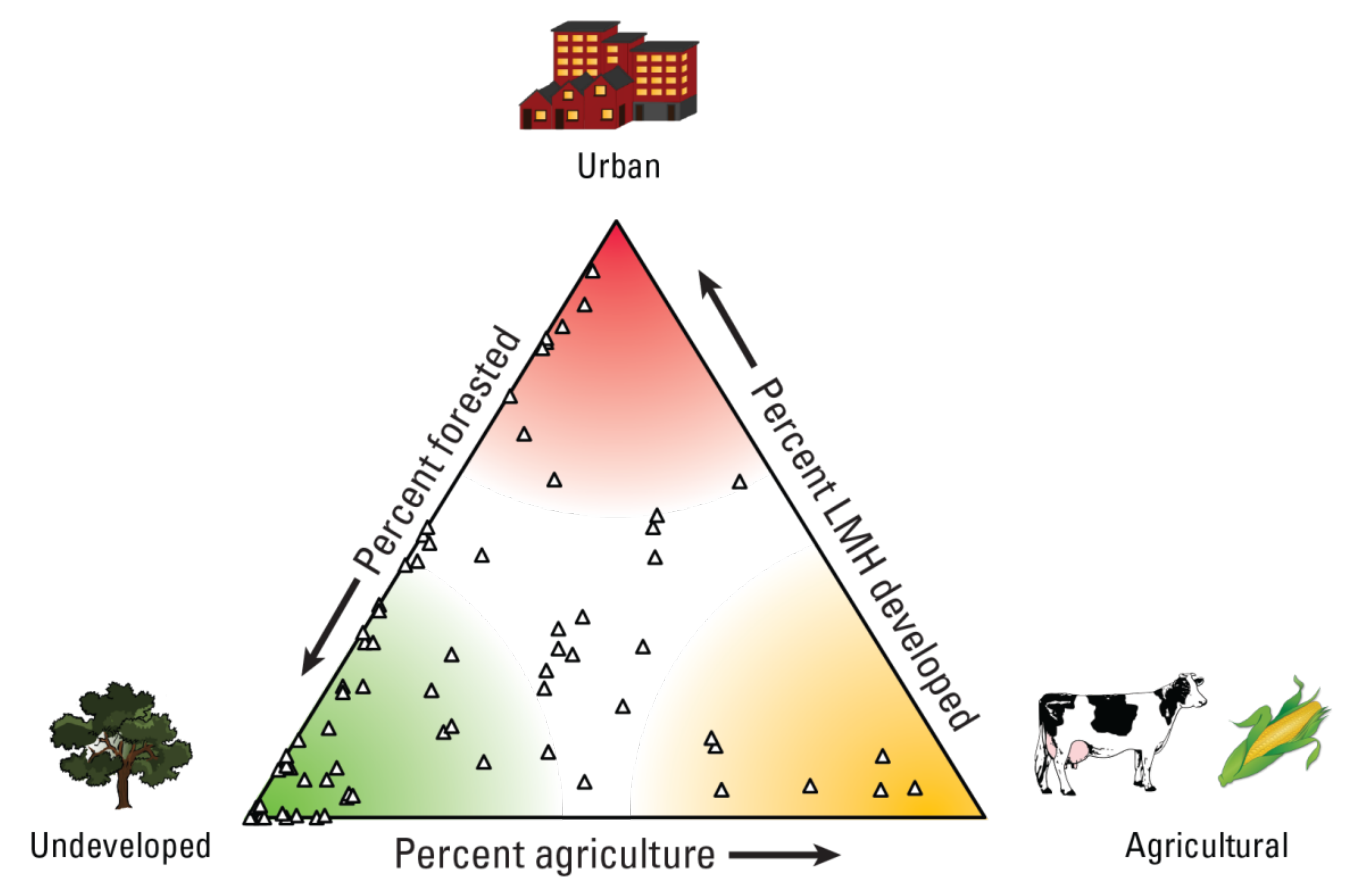

Figure 4. Ternary diagram showing the distribution of percentage of forested, urban (low, medium, and high [LMH] developed), and agriculture land use in study basins of the Pacific Northwest Stream Quality Assessment.

Additional site distribution guidelines included the following:

1. Reference sites were distributed across Oregon and Washington and, to the extent possible, were located near each major urban center in the PNSQA region.

2. Urban tier sites were targeted to capture the complete urban gradient around each of the major urban centers within the region, to avoid having all high or low urban sites in a common metropolitan center.

3. Agricultural sites were targeted in the more intensive growing regions of Oregon and Washington and had low urban development (less than 10 percent).

On the basis of this selection criteria and on desktop reconnaissance with satellite imagery, more than 175 candidate stream sites representing urban tier, reference, or agricultural sites were selected for in-stream reconnaissance conducted by the USGS during autumn and winter 2014. In-stream reconnaissance included observations required for access and safety, and site-type characteristics for the water-sampling location, and to identify a 150-m stream reach where the ecological survey could occur. Information recorded in field notes and photography included (but was not limited to) the location and description of the nearest bridge for water-chemistry sampling, stream-reach wadeability, presence of discharge pipes or point sources in the stream, stream accessibility, streambed substrate, in-stream habitat complexity, and landowner contacts. Field notes and photography were recorded onsite using a tablet-based, electronic field reconnaissance form. Electronic forms from all reconnaissance sites were compiled in spreadsheets for review and final site selection.

On the basis of field reconnaissance results, 88 stream sites were selected -69 sites in the five urban tiers, 8 agricultural sites, and 11 reference sites (table 2; fig. 3). In order to evaluate the effects of hydrologic alteration on stream communities, streamflow or stream-stage data were needed at all sites. Thirty-five of the sites were located at an active USGS or local (county, city) continuous streamgage. Stream stage was monitored at the remaining 53 sites by installing continuous water-level monitoring devices during the PNSQA sampling period. 


\section{Data Collection and Processing}

The 88 stream sites were sampled at various times during April-June 2015, and sampling frequency and timing varied by site type (fig. 5; table 4; appendix A, table A1). Weekly water sampling occurred during a water-quality "index period" that spanned 10 weeks for most sites. This index period was reduced to 4 weeks at all reference sites and at a few of the low urban land-use sites. A one-time ecological survey of stream habitat, algae, benthic macroinvertebrates, and fish was conducted at all sites over a 2-week period, beginning the final week of the 10-week water-quality index period and extending through the week that followed (weeks 10 and 11). Streambed sediment also was collected during the ecological survey for analysis of sediment chemistry and toxicity.

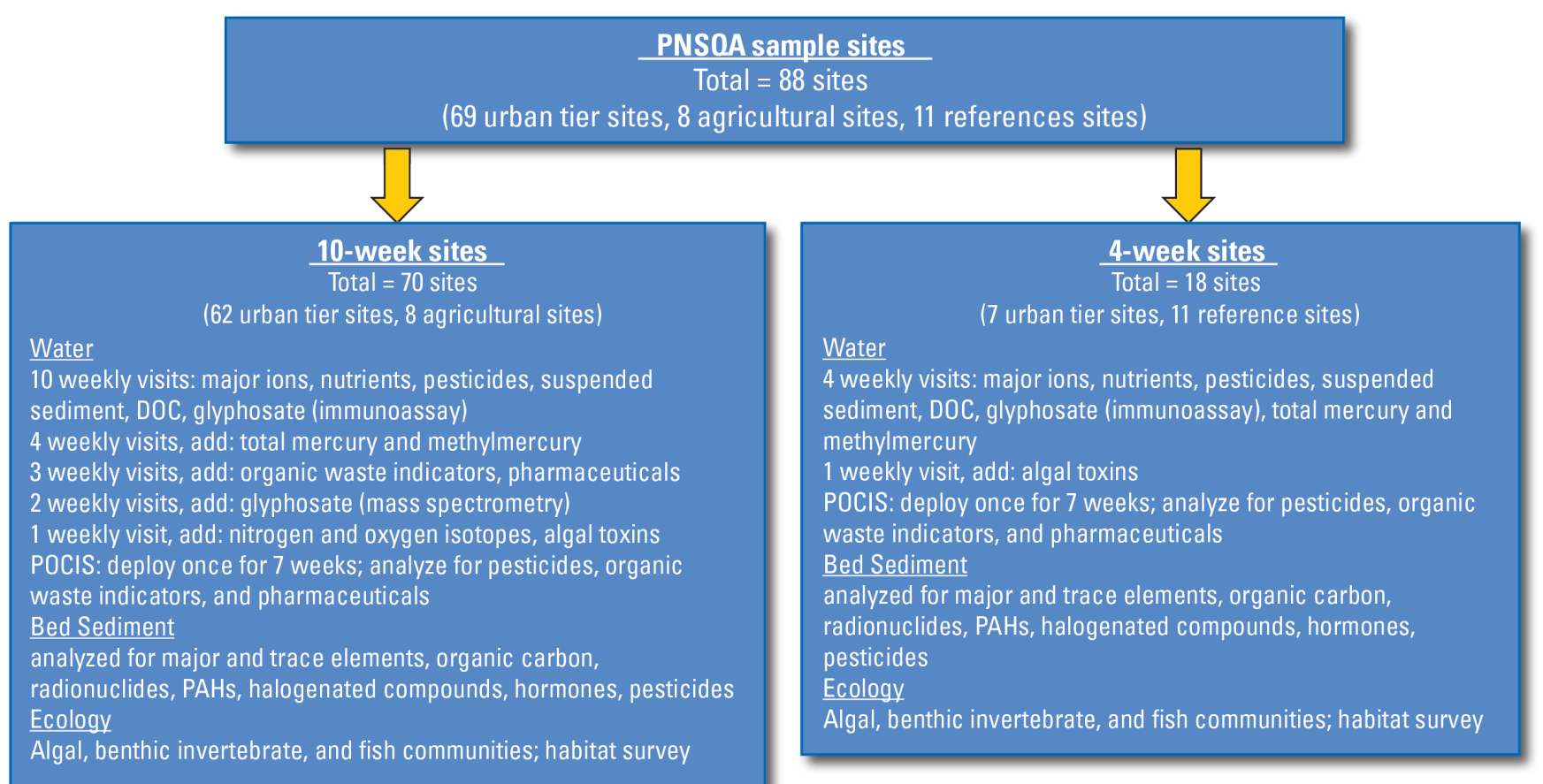

\section{Specialty studies}

Pankow samplers (7 total sites; $2 \mathrm{Ag}, 4$ tier 5 urban): daily and weekly composited pesticide samples

Walling Tubes (13 sites; 1 reference, 4 agricultural, 8 urban tier): sediment analyzed for radionuclides and PAHs(??)

Continuous water quality 6 sites; 1 reference, 2 agriculture, 3 urban tier): continuous temperature, dissolved oxygen, pH, specific

conductance, turbidity, fluorescent dissolved organic matter (FDOM), nitrate, and photosynthetically active radiation (PAR)

Sediment toxicity tests: sediment toxicity of amphipod, midge, and mussels measured at 75 of 88 sites

Figure 5. Flow chart showing sample design for the Pacific Northwest Stream Quality Assessment, 2015. [Ag, agriculture; DOC, dissolved organic carbon; PAHs, polycyclic aromatic hydrocarbons; POCIS, polar organic chemical integrative samplers.]

Data were collected at discrete, integrated, or continuous time frames, depending on the parameter (and stressor) being measured (table 4). Discrete samples that represented conditions at a given date and time were collected on a weekly time interval. Integrated samples represented "average" conditions over the period in which the sampler was deployed. Continuous samples are the parameters measured and recorded at regular time intervals (for example, every 15 or $60 \mathrm{~min}$ ) throughout the 10 week index period or longer. 
Table 4. Matrix of discrete, integrated, and continuous sampling done at each of the Pacific Northwest Stream Quality Assessment sites, 2015.

[Table 4 is a Microsoft Excel ${ }^{\circledR}$ file and is available for download at https://doi.org/10.3133/ofr20171103.]

\section{Water Data Collection}

Weekly discrete samples of water chemistry were collected at all sites. Discrete samples may not accurately reflect the exposure of biota to short-term "spikes" in high chemical concentrations that are potentially acutely toxic. Therefore, to complement weekly collection of discrete stream samples, smallvolume pesticide automated samplers, polar organic chemical integrative samplers (POCIS), and continuous water-quality data also were used in the PNSQA study (table 4).

Use of low-level analytical methods in the laboratory necessitated that water samples be collected using "parts-per-billion" protocols (U.S. Geological Survey, 2006). More than ten 2-person teams were required to complete the water-quality data collection effort. To ensure consistency among the water-quality teams, training for the collection and processing of water-quality samples was provided in March 2015 for all personnel involved with sample collection. Classroom water-quality training was followed by working through all sample and processing procedures in the field prior to the start of the project sampling in April 2015. Detailed sampling timelines, sample parameter matrix, and sample processing steps are summarized in appendix A (tables A1-A4) and briefly described here.

\section{Discrete Water Data Collection}

Discrete water samples were collected using an isokinetic, equal-width increment (EWI) method (U.S. Geological Survey, 2006), where subsamples were collected at 10 increments across the stream using a DH-81 sampler (Davis, 2005). The sampler had a precleaned Teflon ${ }^{\mathrm{TM}}$ cap and nozzle assembly that fit on a 1-L Teflon ${ }^{\text {TM }}$ bottle (U.S. Geological Survey, 2006). Each incremental sample was placed immediately in a precleaned, acid- and methanol-rinsed Teflon ${ }^{\mathrm{TM}}$ churn splitter for compositing prior to processing. In situations where stream conditions did not meet the requirements for collection of a representative EWI sample (velocity less than $1.5 \mathrm{ft} / \mathrm{s}$ and [or] depth less than $1 \mathrm{ft}$ ), multiple vertical samples were collected across the stream width or a grab sample was collected from the centroid of flow (see appendix A, table A4, for stream conditions for each type of sample collection). The Pacific Northwest had a drought in 2015, with no rain measured during the study in most of the region. As a result, grab samples were collected from many of the streams. All field equipment was cleaned prior to sampling according to USGS protocols (Wilde, 2004) and rinsed with native water immediately before samples were collected.

Samples were collected and processed using standard USGS protocols described in the National Field Manual (Wilde and others, 2004; appendix A, table A4) and briefly described here. For EWI and multiple vertical samples, all raw (unfiltered) sample bottles were filled first while the composite sample was continuously churned within the churn splitter. Next, churning was stopped and all filtered ( 0.45 - $\mu$ m capsule filter) samples were collected from the churn splitter. Samples for organic compounds were filtered using a $10 \mathrm{~mm}$ syringe equipped with a $0.45-\mu \mathrm{m}$ syringe filter from a $500 \mathrm{~mL}$ baked amber glass bottle that was filled during the first processing step (filling of raw sample bottles). After all sample bottles were filled, those requiring preservatives were preserved in a processing chamber located within each water-quality vehicle. For those sites where grab samples were collected, samples were collected directly from the stream in sample bottles for unfiltered samples, and in a precleaned 1-L or 3$\mathrm{L} \mathrm{Teflon}^{\mathrm{TM}}$ sample bottle for filtered constituents. Grab samples were processed as described for EWI samples except that filtered parameters were collected from the 1-L Teflon ${ }^{\mathrm{TM}}$ bottle, not a churn splitter. 
Field properties of specific conductance, $\mathrm{pH}$, dissolved oxygen, and water temperature were measured at the time of sampling with a field-calibrated multiparameter sonde (Wilde, various dates). Field records (either paper forms or electronic field forms) for all water-quality sampling events were maintained that included the site name, date and time of sample collection, medium code, field parameters (temperature, dissolved oxygen, $\mathrm{pH}$, specific conductance), lot numbers for filters and field preservatives, and general observations (weather, streambed substrate, streamflow conditions, and water clarity). Bar codes were affixed to each sample bottle and field form as an auxiliary data match and tracking method. After field processing (steps described in appendix A, table A4), all water-quality samples were stored appropriately (refrigerated or frozen) until shipment to the receiving laboratory.

At all urban tier 2-5 sites, 5 of 12 urban tier 1 sites, and all agricultural sites, discrete water samples were collected weekly during a 10-week water-quality index period from mid-April to midJune 2015 (70 sites). The 11 reference sites and 7 of the 12 urban tier 1 urban sites were only sampled during the final 4 weeks of the water-quality index period (18 sites). Because many of the urban tier 1 sites had low urban land cover in their watersheds, the urban tier 1 sites were split between 10- and 4week sampling schedules to accommodate sample and laboratory budget constraints. The decision to move an urban tier 1 site to a reduced sample schedule was based on best professional judgment by using GIS data and examining the watersheds in Google Earth ${ }^{\mathrm{TM}}$ to identify sites that were at the lowest end of the development gradient and that did not appear to have much or any development in the lower watershed near the sampling location. These urban tier 1 sites were identified as having near-reference conditions, so consequently they were sampled only four times to match the sampling schedule for the reference sites and conserve costs.

Weekly water-quality samples were collected for major ions, nutrients, pesticides, glyphosate, and suspended-sediment concentration (fig. 5; Table 4). A suite of other water-quality parameters was collected on a reduced schedule during the water-quality index period: (1) Isotopes of nitrate $\left({ }^{15} \mathrm{~N}\right.$ and

${ }^{18} \mathrm{O}$, one time), (2) organic wastewater indicators and pharmaceuticals (three times), and (3) algal toxins (one time) (appendix A, table A3). Ultra-clean trace-level sampling procedures and equipment were used to collect surface-water samples for low-level total mercury and methylmercury analysis (U.S. Environmental Protection Agency, 1996; Lewis and Brigham, 2004) four times at each site as a grab sample from the centroid of flow at about $0.3 \mathrm{~m}$ below the water surface using a Teflon ${ }^{\mathrm{TM}}$ bottle. For the 10-week sites, samples for mercury analysis were collected about every 3 weeks; for the 4-week sites, samples were collected for all 4 weeks. The collected sample was acidified immediately with ultra-pure hydrochloric acid (Lewis and Brigham, 2004).

\section{Integrated Water Data Collection}

Passive samplers were used to collect time-weighted average concentrations of polar organic compounds at 75 of the 88 sites. At four urban tier 5 sites and three agricultural indicator sites, smallvolume pesticide automated samplers were deployed to evaluate daily and weekly changes in pesticide concentrations. 
Polar Organic Chemical Integrative Samplers (POCIS)

The POCIS are designed to accumulate organic compounds with moderate-to-high water solubility (polar or hydrophilic) from surface water, and were deployed at 75 sites during the PNSQA data collection period. The POCIS consists of a solid material (sorbent) contained between two microporous membranes that allow water and dissolved chemicals to pass through to the sorbent (fig. 6). The POCIS allow estimation of time-weighted average concentrations for many chemical stressors (pharmaceuticals and pesticides) for ecological risk assessment. POCIS deployment lasted 5-6 weeks, from the week of May 4 or 11 until the week of June 15, 2015.
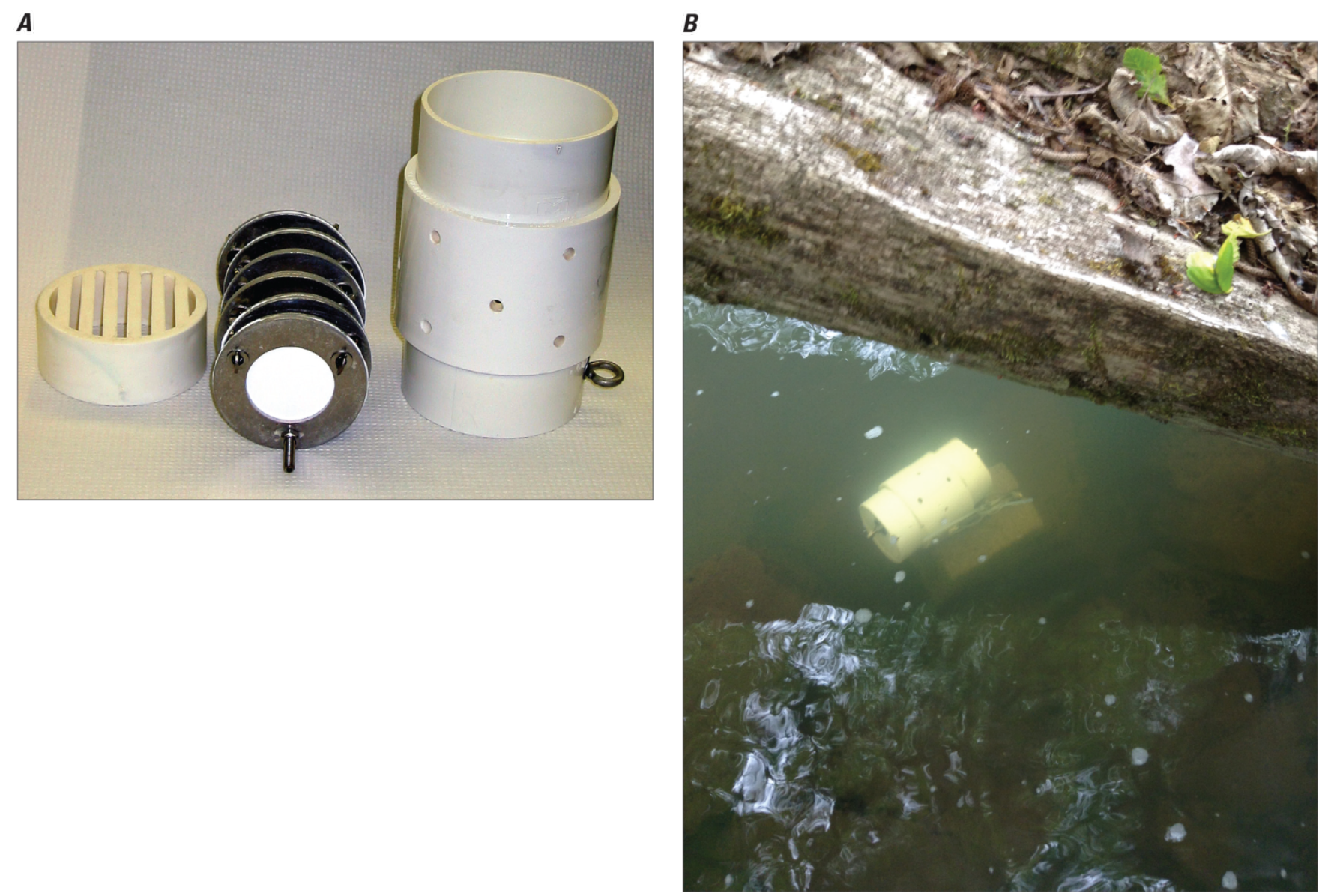

Figure 6. Photographs showing $(A)$ polar organic chemical integrative sampler (POCIS) disks mounted in stainless steel rack and example of protective deployment canister for the field, and $(B)$ deployed POCIS on cinder-blockand-cable infrastructure in Whipple Creek, Washington, 2015. Photograph $A$ from Alvarez (2010), photograph B by Danial Polette, U. S. Geological Survey, May 2015. Note: For the current study, only four POCIS disks were used per canister, not six as shown in photograph $A$. 
Field deployment followed the guidelines provided in Alvarez (2010). Successful deployment required a stream location with sufficient depth so that the sampler would remain submerged during the deployment period and would be protected from excessive sediment accumulation, debris, and vandalism. Effective anchoring systems were adopted on the basis of site-specific characteristics (for example, sandy compared to rocky substrate, streamflow variability, and so forth). Field records were maintained that included the site name, date and time of deployment and retrieval, and observations of streambed substrate, streamflow conditions, and water clarity.

About 10 percent of the POCIS were accompanied by field blanks used to assess any accumulation of target and non-target compounds from the air during shipment and transport. The POCIS field-blank protocol specified that the blank canisters be open to the air at the same time and place as the field POCIS were exposed to air during deployment and retrieval. Between deployment and retrieval of the field POCIS, the POCIS blank canisters were kept sealed and stored from -20 to $0{ }^{\circ} \mathrm{C}$. All field POCIS and blank canisters were stored on ice during transport to and from the field location. After the deployment period, deployment canisters containing the POCIS samplers, field blanks, and log sheets were stored at -20 to $0{ }^{\circ} \mathrm{C}$ until shipment to the USGS Columbia Environmental Research Center (CERC) for analysis.

\section{Small-Volume Pesticide Automated Samplers}

Small-volume pesticide automated samplers (pesticide autosamplers) were used to help determine if increased sampling frequency would more accurately characterize in-stream pesticide stressor conditions experienced by biota, including short-lived but acutely toxic events. Pesticide autosamplers were designed and built at Portland State University to collect fixed-point, low-volume, filtered samples for the analysis of pesticides in surface water (fig. 7). The pesticide autosamplers were deployed at seven PNSQA sites to collect daily and weekly composite water samples for pesticide analysis during the 10-week water-quality index period (table 4). The seven locations were:

- Two urban sites (Longfellow and Thornton Creeks) in Seattle, Washington, and two urban sites (Kellogg and Fanno Creeks) near Portland, Oregon; and

- Two agricultural sites (Bertrand and Fishtrap Creeks) in northern Washington and one agricultural site (Zollner Creek) in the north-central Willamette Valley, Oregon.

The pesticide autosamplers were programmed for unattended collection of stream water aliquots over a 1-week period. A filtered aliquot of stream water is collected every 6 hours in daily composite vials (4 aliquots per vial, with the "day" typically starting at noon) and every 12 hours in the weekly composite vial. Thus, eight vials are used for seven daily samples and a one weekly sample. A ninth vial containing a known pesticide spike mixture in native stream water also was included to assess the potential for compound degradation during the weekly collection period. A 6-mL aliquot of a 1:1 methanol-water mixture was added to all nine vials before deployment as a preservative. Prior to deployment, each vial was labeled with the station identification number, vial number, date, and initial weight. Two autosamplers were available for each of the seven sites being sampled so that a weekly unit exchange could be made. This arrangement minimized interruption by allowing ample time to remove and replace sample vials, charge batteries, clean tubing, and replace consumable components such as filters. Sample vials were shipped on ice overnight to National Water Quality Laboratory (NWQL, Denver, Colorado) every week during the study. 


\section{A}

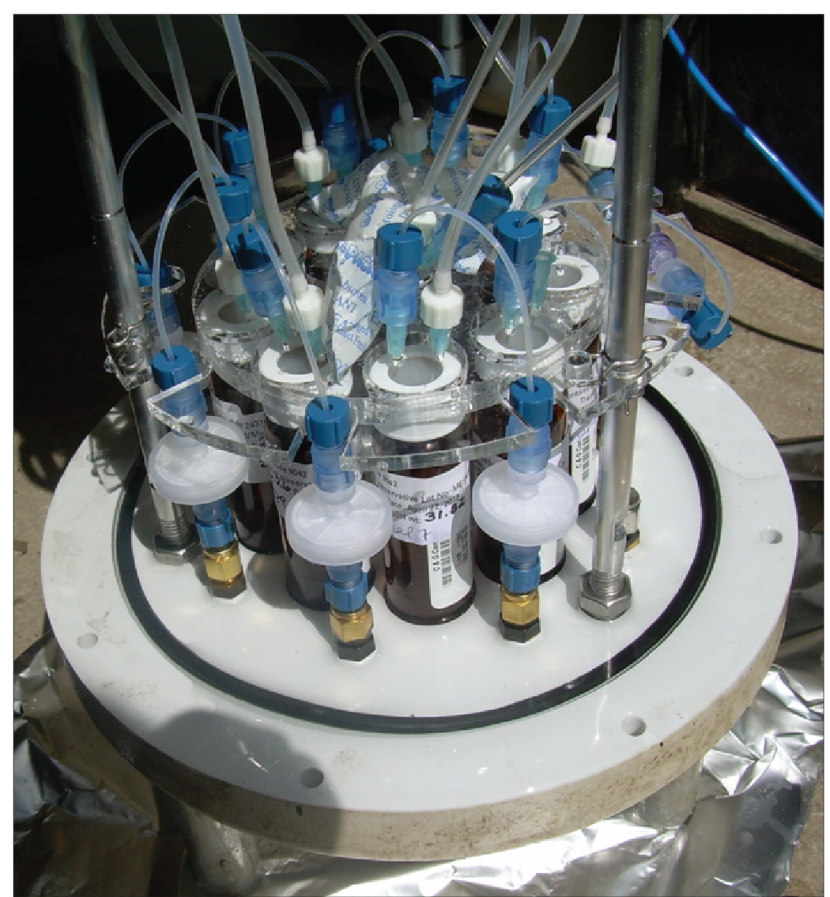

$c$

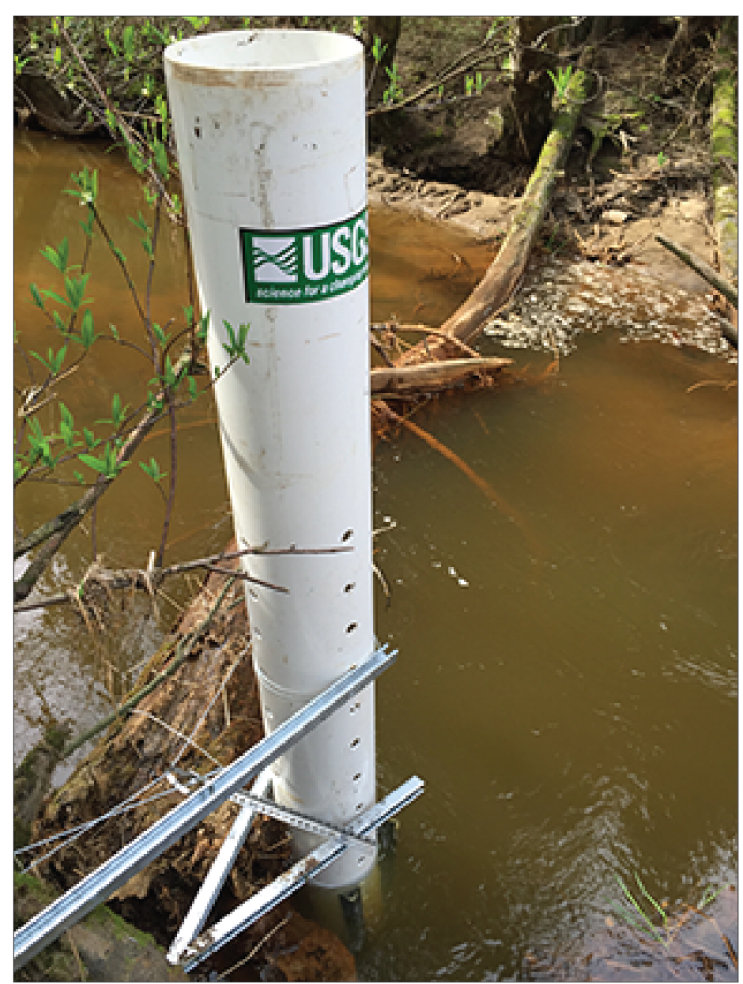

$B$

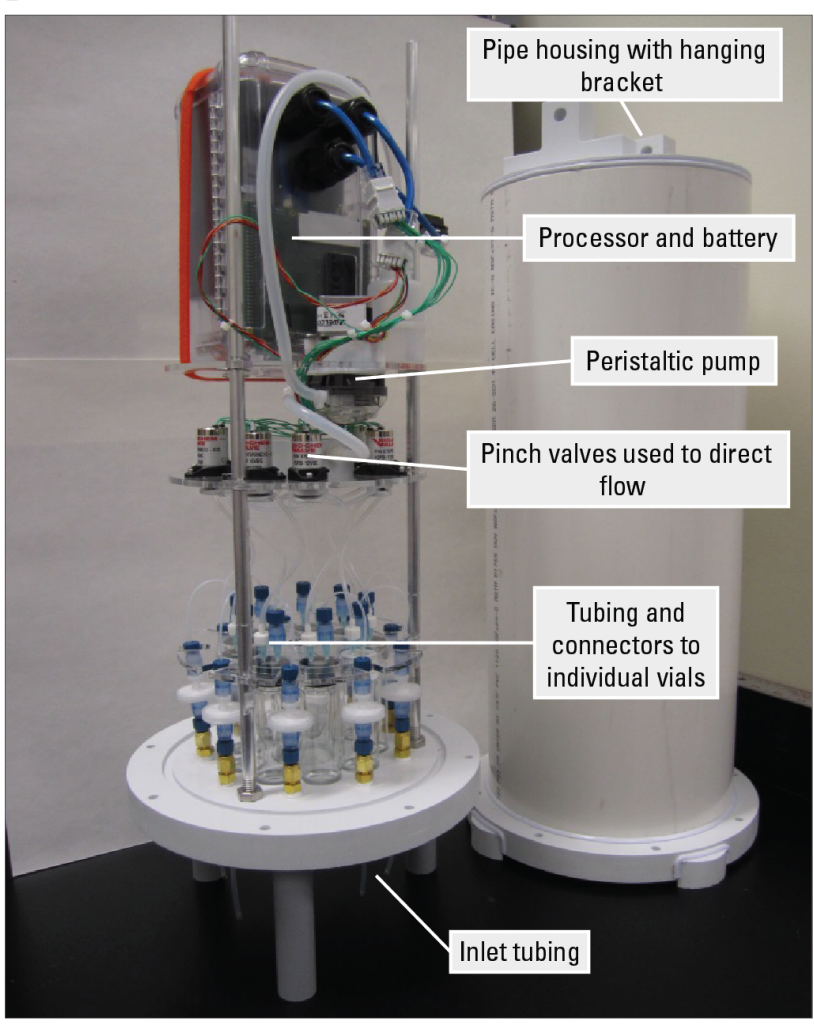

D

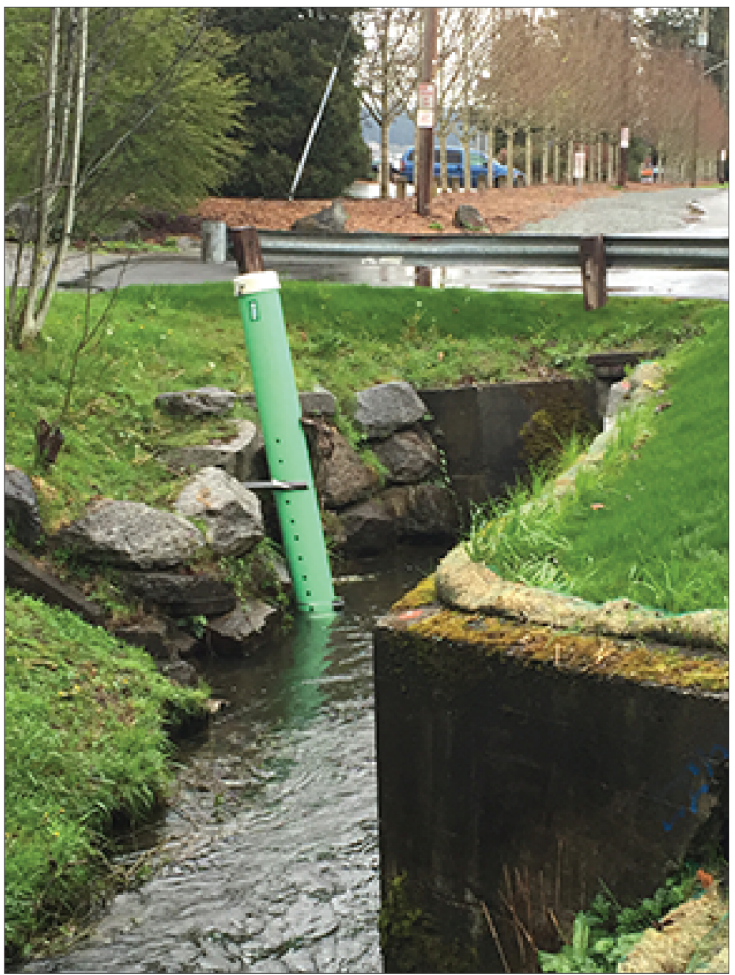

Figure 7. Photographs showing small-volume pesticide automated samplers $(A$ and $B)$ used to collect filtered water at sub-daily intervals for pesticide analysis as part of the Pacific Northwest Stream Quality Assessment, and examples of field deployments at Bertrand $(C)$ and Thornton Creeks $(D)$, Washington, 2015. Photographs $A$ and $B$ from Journey and others (2015). Photographs C and D by Richard Sheibley, U.S. Geological Survey, April 2015. 


\section{Continuous Water Data Collection}

\section{Water Temperature and Water Level Monitoring}

Digital temperature-data loggers were used to continuously monitor water temperature at all stream sites in the PNSQA study. Internally logging digital devices were deployed prior to April 15, 2015, the beginning of the data collection period, and remained deployed until September-October 2015 , recording temperature at 1 -h intervals. When possible, loggers were deployed about $15 \mathrm{~cm}$ above the streambed, out of direct sunlight, and attached to rebar anchored in the streambed or to stable parts of streamgage infrastructure or bridge piers if these structures were present at the site.

Specifications for the HOBO ${ }^{\circledR}$ Water Temp Pro v2 U22 loggers used in the PNSQA study are provided in appendix A (table A5). Guidance from the manufacturer and U.S. Forest Service concerning the deployment, calibration, and maintenance generally was followed for the PNSQA study (Onset Computer Corporation, 2012; Dunham and others, 2005).

At stream sites where continuous streamgages did not exist (53 of 88 sites; table 4$)$, digital water-level loggers were deployed that recorded water temperature and water level (stage). Internally logging digital devices were deployed prior to April 15, 2015, the beginning of the data collection period, and remained deployed until September-October 2015. Specifications for the HOBO ${ }^{\circledR}$ U20001-04 digital water level loggers that were used are provided in appendix A (table A5). Guidance from the manufacturer and the USGS for deployment, calibration, and maintenance generally was followed for the PNSQA study (Sauer and Turnipseed, 2010; Onset Computer Corporation, 2014). Deployment included the installation of two loggers per site-one to measure water level (water column) and one to measure barometric pressure (air). Each water logger was mounted at a depth where it would remain continually submerged, yet be readily accessed if needed. The barometric pressure logger typically was fixed to a nearby tree or bridge infrastructure.

After installation, a reference point (RP) was established on a permanently fixed structure, such as a fencepost or mark on a bridge; the distance from this RP to the water surface was measured to verify that the water-level logger had not moved while it was deployed. Guidelines for establishing the RPs are as follows:

1. An arbitrary datum that would cover all low stages (to ensure no negative numbers) was selected. For example, if the maximum depth of a cross section is $5 \mathrm{ft}$, and the lowest RP is $0.5 \mathrm{ft}$ out of the water, a datum number of $6.0 \mathrm{ft}$ would be selected for a lower RP.

2. The water-level logger and RPs were located in the same stream pool with the same control conditions.

Water-level and barometric-pressure loggers were programmed to record on 15-min intervals for the duration of the study. For the most part, water-level loggers were installed and not moved until the study was over. In situations where water-level loggers had to be moved (for example, the stream was going dry or data were downloaded), the distance from the RP to water surface was measured prior to removing the logger and immediately after it was redeployed to correct for any difference in watercolumn depth that may have occurred to the logger. Additionally, measurements from the RP to water surface were made each time the water quality crew visited the site, so that these values could be used to check the data for consistency and quality. 
Continuous Water Quality and Algal Biomass

An intensive investigation of water quality and associated benthic algal biomass was conducted at six sites in the PNSQA region to provide high temporal resolution information on general water quality and nutrient dynamics (table 4). The six sites (4 in Washington and 2 in Oregon) included urban and agricultural sites and were selected to include a range of expected nutrient conditions. The sites were instrumented for 6 months (March-September 2015) to measure continuously (15-min intervals) the following parameters:

- Temperature;

- Dissolved oxygen;

- $\mathrm{pH}$;

- Specific conductance;

- Fluorescent dissolved organic matter (FDOM - a proxy for carbon concentrations) and turbidity, using an $\mathrm{EXO} 2^{\circledR}$ (YSI Incorporated);

- Nitrate, using a SUNA V2 (Submersible Ultraviolet Nitrate Analyzer, Sea-Bird Scientific); and

- Streamside photosynthetic light (photosynthetically active radiation, PAR) meter (Onset). During monthly site visits, reach-scale benthic-algae chlorophyll samples were collected, and measurements of aquatic macrophyte cover and percentage of canopy cover were made using the transect methods described in Fitzpatrick and others (1998). In addition to the weekly water-quality sampling during the index period (April-June), nutrient samples also were collected monthly at these six sites in March, July, August, and September 2015.

\section{Sediment Data Collection}

During the PNSQA study, suspended-sediment concentration, suspended-sediment chemistry, and streambed-sediment chemistry data were collected using discrete and time-integrated methods.

\section{Discrete Sediment Data Collection}

Weekly discrete samples of suspended-sediment concentration were collected at each site from the EWI or grab samples as described earlier and sent to the USGS Cascades Volcano Observatory. At the onset of the ecological survey before any biological samples were collected, streambed-sediment samples were collected once at all PNSQA sites using established USGS protocols (Shelton and Capel, 1994; Radtke, 2005). Four inch (about-10 cm) diameter stainless-steel cylinders and stainless-steel spatulas were used to collect the streambed material. Multiple collections of sediment were made at depositional areas along the 150-m ecological assessment reach, targeting locations where fine-grained sediments accumulated. Depositional zones across the study reach were sampled in approximate proportion to their bottom surface area in the reach. Samples were not sieved, but material larger than about $2 \mathrm{~mm}$, such as gravel, sticks, or debris, were removed by a gloved hand. The collection method required pushing the stainless-steel cylinder into the streambed to a depth of $2 \mathrm{~cm}$, then sliding the spatula under the cylinder to support the enclosed streambed core. Each streambed core was lifted gently out of the water to minimize the loss of fine material and all cores were composited in a large plastic bucket. 
About 6-10 L of streambed material were collected along the ecological assessment reach. Samples were homogenized briefly immediately upon collection by mixing with a clean stainless steel spoon or with a precleaned stainless steel paint mixing attachment and a cordless drill. The bulk sediment sample was immediately placed on ice and shipped to CERC (Columbia, Missouri), where samples were refrigerated in the dark at $4{ }^{\circ} \mathrm{C}$. Prior to further analysis and testing, samples were homogenized using a power mixer and then divided into aliquots for analyses of various chemical constituents and for bioassays (toxicity testing). Analyses were conducted for organic wastewater indicators, polycyclic aromatic hydrocarbons (PAHs) and other semi-volatile compounds, current use and organo-chlorine insecticides, polychlorinated biphenyls (PCBs), polybrominated diphenyl ethers (PBDEs), hormones, major and trace elements, organic carbon, and grain size. Toxicity testing was conducted on three aquatic organisms by using replicate beakers filled with the homogenized sediment.

\section{Integrated Sediment Data Collection}

Suspended-sediment samples were collected from 13 streams using time-integrating passive samplers referred to as a Walling tubes (Phillips and others, 2000; Gellis and others, 2016) to assess sediment sources using major and trace elements and radionuclides. The Walling tube sampler was made from commercially available PVC pipe (98- $\mathrm{mm}$ inner-diameter) cut to a length of about $1.0 \mathrm{~m}$ and attached to metal posts driven into the streambed (fig. 8). The facing upstream was affixed with a funnel that had a 4-mm nozzle, and the downstream end of the tube had an endcap with a hole drilled in the center to allow a 4-mm plastic tube to be inserted. By design, as water passes into the tube through the small opening in the funnel stem, the velocity decreases in the larger-diameter tube, allowing suspended sediment to settle. Typically four tubes were deployed within the $150-\mathrm{m}$ reach of a site, at locations of moderate to high velocity, where two tubes were installed at each of two locations by placing one tube above the other on the metal posts (fig. 8). To ensure that samples consisted of suspended sediment and not bed sediment, the bottom tube was submerged at least $15 \mathrm{~cm}$ above the channel bed. However, if base flows were especially low at the time of deployment, the top tube might be out-of-water, but in those cases, these tubes would collect sediment samples at higher flows.

Suspended-sediment collection by Walling tubes was conducted from March through September 2015. Because of low sediment concentration and unusually dry conditions, the tubes were inspected at a set time interval (every 3-4 weeks) so that sufficient sediment could accumulate for analyses of major and trace elements and radionuclides To collect the sediment samples, the tubes were removed from their posts, the end caps were opened, and the water and sediment were poured into a 5-gal plastic bucket. A spray bottle filled with deionized water was used to rinse any remaining sediment from the tubes. After the samples were collected, the tubes were cleaned with a brush and deionized water, then rinsed with native water. The water-sediment mixture in the 5 -gal plastic bucket was stored on ice or in a cooler prior to final laboratory preparation. Date and time of each deployment and retrieval were recorded in field sheets. 


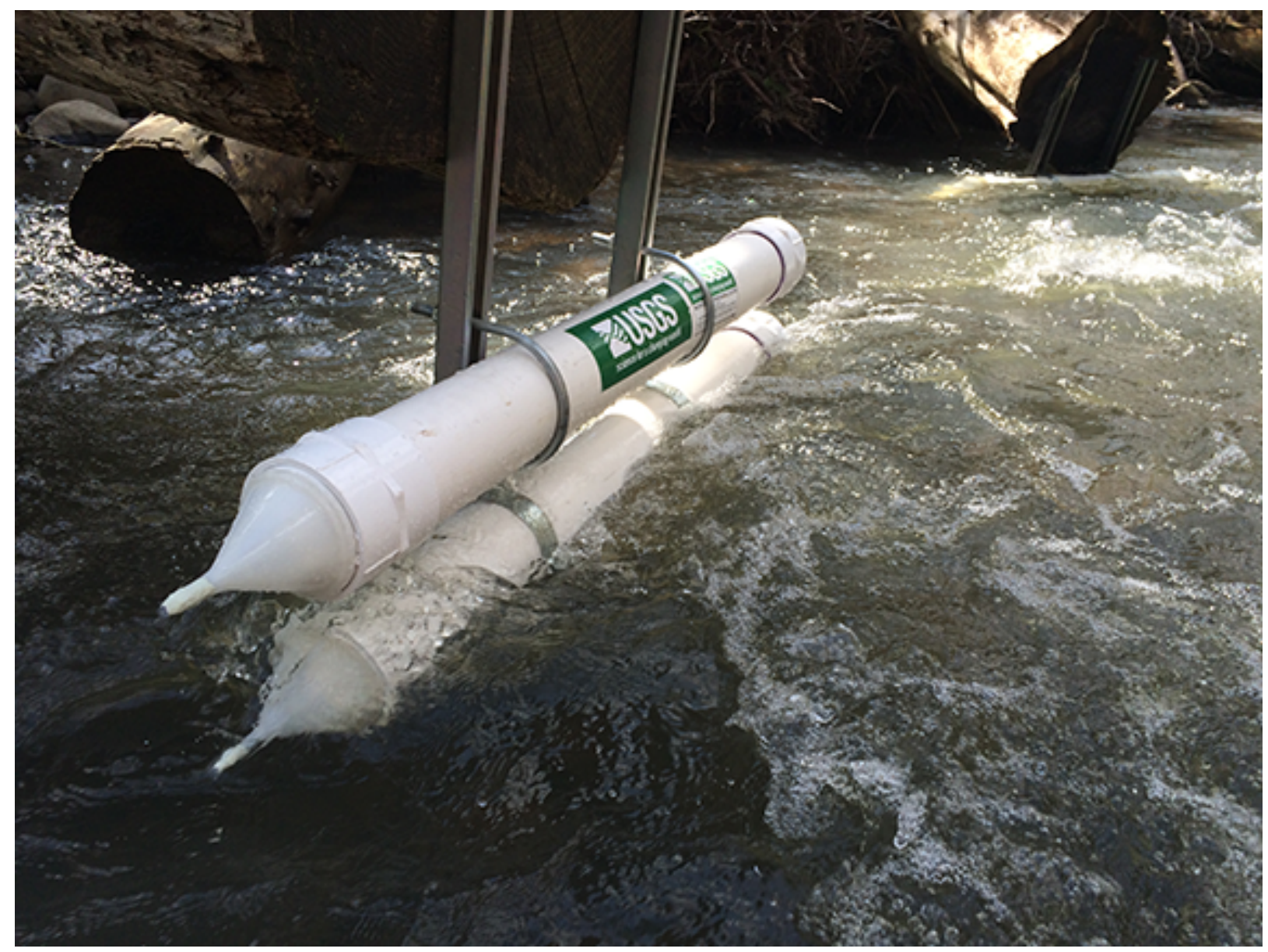

Figure 8. Photograph showing Walling tubes installed to collect suspended sediment in Rock Creek near Damascus, Oregon. Photograph by Steven Sobieszczyk, U. S. Geological Survey, May 2015.

\section{Ecological Data Collection}

Six teams of six people each were deployed during an approximately 10-day period in mid-June at the end of the water-quality index period to sample all 88 sites. To ensure consistency in collecting the biological community samples and conducting the physical-habitat surveys, the teams were trained during the spring of 2015 on the methods described in USGS ecological sampling and bed-sediment collection protocols. Algae, invertebrate, and fish-community samples were collected and habitat was assessed along a 150-m ecological-assessment reach, according to the methods described in Moulton and others (2002). All field data were recorded on electronic forms using hand-held tablet computers. Raw field data collected from the fish and habitat surveys, and field records for the algal and invertebrate samples destined for laboratory analysis were loaded in the USGS BioData biological database (https://aquatic.biodata.usgs.gov).

Algal and invertebrate communities were sampled using standard USGS richest-targeted habitat (RTH) protocols (Moulton and others, 2002; Hambrook and Canova, 2007). RTH samples are intended to represent the habitat features (usually a riffle) having the greatest diversity of organisms within a given stream reach. The benthic algae were scraped from natural substrates (for example, flat rocks) to obtain a targeted area of $150 \mathrm{~cm}^{2}$. The substrate was scraped with a brush in a defined area and flushed 
into a 500-mL bottle with native water. Typically, 11 subsamples of equal size were combined in a single composited algal sample to represent the site. From the composite sample, two aliquots were removed and filtered in the field for later analysis at the NWQL of chlorophyll $a$ and ash-free dry mass. Two additional aliquots were filtered as backups in the event of sample loss or damage. The remainder of the sample was preserved with buffered formalin at a concentration of about 5 percent and sent to the University of Colorado for algal taxa identification and enumeration.

Invertebrate samples were collected from the RTH (typically a rock riffle), using a modified Surber sampler with $500-\mu \mathrm{m}$ mesh net that samples a $0.25 \mathrm{~m}^{2}$ area of substrate (Moulton and others, 2002). The total invertebrate sample area was targeted at $12,500 \mathrm{~cm}^{2}\left(1.25 \mathrm{~m}^{2}\right)$, the sum of a composite of five modified Surber samples. The samples were collected on a 500- $\mu \mathrm{m}$ sieve and large organic and inorganic debris was removed, and then transferred to a 1-L bottle and preserved with 10-percent buffered formalin. Large or rare invertebrates, such as crayfish and larger mollusks, were photographed and released in accordance with collection permit procedures. Invertebrate samples were shipped to NWQL for identification and enumeration of taxa (generally either genus or species).

A representative fish-community sample was collected at each site using backpack-mounted electrofishing units. In streams wider than about $10 \mathrm{~m}$, two backpack units were used in tandem to cover a larger area of the stream and to increase efficiency. Two electrofishing passes of the sampling reach were conducted using the same number of backpacks in each pass. Fish generally were identified to species, counted in the field, and then released downstream of the collection reach. In the few cases where a fish could not be positively identified in the field, an individual fish was preserved for later identification. At a limited number of sites, a small number of freshwater sculpin (Cottus sp.) and (or) juvenile salmonids were collected for additional genetic analysis of selected tissues.

The physical habitat of the reach was characterized following USGS protocols (Fitzpatrick and others, 1998). Reach lengths for this study were $150 \mathrm{~m}$ at each site. Qualitative and quantitative measurements were collected at 11 primary and 10 secondary transects. Depending on the transect type, these measurements included, but were not limited to, depth, wetted width, substrate particle size, canopy cover, macrophyte coverage, bank height, presence of bars and islands, and in-stream fish habitat. Geomorphic channel units such as pools, riffles, and runs were characterized by size and frequency.

\section{Laboratory Analyses}

Most of the laboratory analyses of water, sediment, and invertebrate samples were conducted by the NWQL (appendix B, tables B1-B11), and the methods are briefly described here.

\section{Discrete Water Samples}

All water samples collected for major ions, nutrients, and pesticides from the 10-week and 4week sites were analyzed by the NWQL (tables B-1 through B-3, respectively). Total phosphorus (TP) concentrations were determined by colorimetry according to Environmental Protection Agency (EPA) method 365.1 (O’Dell, 1993), whereas dissolved ammonia, nitrite, and orthophosphate colorimetric analyses were described by Fishman (1993). Dissolved nitrate-plus-nitrite concentrations were determined by low-level enzyme reduction colorimetry using an automated discrete analyzer, as described by Patton and Kryskalla (2011). Concentrations of dissolved cations were determined by inductively coupled plasma-atomic emission spectroscopy (ICP/AES) (Fishman, 1993), and concentrations of dissolved anions were determined by ion chromatography, as described by Fishman and Friedman (1989). 
Pesticides were analyzed using direct aqueous injection liquid chromatography tandem mass spectrometry (DAI LC-MS/MS) (appendix B, table B3; Sandstrom and others, 2015). The pesticide analytical method quantified 229 pesticides and pesticide degradates in filtered water samples (appendix B, table B3; Furlong and others, 2014; Sandstrom and Wilde, 2014; Sandstrom and others, 2015). The targeted pesticides represent a broad range of chemical classes and were selected on the basis of criteria such as current use intensity, probability of occurrence in streams and groundwater, toxicity to humans or aquatic organisms, and precision of analytical methods. The method uses direct injection of a $100-\mu \mathrm{L}$ sample onto the LC-MS/MS without any sample preparation other than filtration. Samples were analyzed with two injections, one in electrospray ionization (ESI) positive mode and one in ESI negative mode, using two multiple reaction monitoring transitions for each analyte. Recoveries for most analytes ranged from 80 to 120 percent in the water types tested, with relative standard deviations of less than 30 percent. The method detection limits (MDLs) ranged from 1 to $106 \mathrm{ng} / \mathrm{L}$ for all but six analytes that had MDLs from 250 to $1,000 \mathrm{ng} / \mathrm{L}$.

Using a method similar to that used for pesticides, 112 human-use pharmaceuticals were analyzed using DAI LC-MS/MS (Furlong and others, 2014). This method uses DAI LC to separate individual pharmaceuticals and is coupled to a triple quadrupole mass spectrometer (MS) using an electrospray ionization source operated in the positive ion mode. The retention time of each analyte from the LC and two unique precursor ion/product ion pairs produced during ionization form the basis for identification. Quantitation is based on the internal standard method using 19 isotopically labeled pharmaceutical and atrazine analogues. For calibration, a minimum of 5 and as many as 15 calibration levels, specific to each compound in the method, are used to encompass the range of expected responses during analysis.

Sixty-nine wastewater indicator compounds indicative of domestic and industrial wastewater were determined in 1-L whole-water samples using liquid-liquid extraction and gas chromatographymass spectrometry (GC/MS) (Zaugg and others, 2006a). Briefly, 1-L whole water samples were treated with $10 \mathrm{~g}$ of sodium chloride and extracted using continuous liquid-liquid extraction using methylene chloride. The methylene chloride extract was concentrated, internal standards were added, and then the extracts were reduced to a final volume of $400 \mu \mathrm{L}$. The sample extracts were analyzed by capillary gas chromatography/mass spectrometry using temperature programming to achieve chromatographic separation and electron impact ionization at 70 electron volts. The mass spectra were collected under full-scan conditions. Compound identification was by comparison of chromatographic retention time and sample mass spectra to library spectra from standard compounds analyzed under identical conditions. Quantitation was by the internal standard method. Full details of the procedure are available in Zaugg and others (2006a).

Stable isotopes of nitrogen $\left({ }^{15} \mathrm{~N}\right)$ and oxygen $\left({ }^{18} \mathrm{O}\right)$ of nitrate were analyzed once at each of the 10-week sites (appendix A, table A3). Samples were filtered in bottles and frozen until nitrate concentration data were received and then shipped to the USGS stable isotope laboratory in Reston, Virginia. Analysis of nitrate isotopes were made following the method of Coplen and others (2012). Dissolved nitrate in water is converted to nitrous oxide by denitrifying bacteria, and the nitrous oxide is analyzed for nitrogen and oxygen isotopic abundance by continuous-flow isotope-ratio mass spectrometry.

Composited water samples were analyzed approximately monthly (three times; table A3) for pharmaceuticals by DAI LC-MS/MS (appendix B, table B4; Furlong and others, 2014) and for organic wastewater indicator compounds by GC/MS (appendix B, table B5; Zaugg and others, 2006a), and at each of the 10-week sites. Pharmaceutical samples were syringe-filtered into $40-\mathrm{mL}$ vials and organic waste indicator samples were collected as whole water samples in a 1-L baked amber glass bottle. Compounds were identified using strict identification criteria, which included analyzing standard 
reference materials and comparing retention times and relative ratios of the mass spectrum. Compounds were quantitated using internal standard procedures. Concentrations determined to be less than the lowest calibration standard were qualified to signify the lower confidence in the extrapolated concentration. Compounds were not quantitated and were reported as not detected if they did not strictly adhere to MS identification criteria. Compounds identified with concentrations within the calibration range were reported without qualification unless quality control or holding times were compromised. Compounds with concentrations greater than the highest calibration standard were diluted to within the calibration range and reanalyzed.

About every 3 weeks at the 10-week sites and weekly at the 4-week sites (four times total at all sites), samples were analyzed for methylmercury by gas chromatographic separation with cold vapor atomic fluorescence spectrometry (De Wild and others, 2002) and for total mercury by oxidation, purge and trap, and cold vapor atomic fluorescence spectrometry (Method 1631, revision E; U.S. Environmental Protection Agency, 2002) at the USGS Wisconsin Mercury Research Laboratory (WMRL) in Middleton, Wisconsin. The WMRL also analyzed all samples collected at the 10-week and 4-week sites for dissolved organic carbon and ultraviolet absorbance at 254 nanometers (nm).

Weekly composited filtered-water samples were analyzed for glyphosate using an enzymelinked immunosorbent assay (ELISA) at the USGS Texas Water Science Center (Mahler and others, 2017). Glyphosate also was sampled twice (during weeks 2 and 10) at all sites and analyzed at the Kansas Organic Geochemistry Research Laboratory (OGRL) using an online solid-phase extraction and liquid chromatography/tandem mass spectrometry technique (Meyer and others, 2009). The data from this method were used to evaluate quality control on the data analyzed by the ELISA method.

A separate water sample was collected at all 88 sites during week 10 to survey across the region for the presence of algal toxins (microcystin) in streams. These samples were prepared and analyzed at the Kansas OGRL following methods outlined in Loftin and others (2016). Unfiltered samples were lysed by three sequential freeze-thaw cycles at -20 and $25^{\circ} \mathrm{C}$ and then were syringe-filtered through 0.7 $\mu \mathrm{m}$ glass fiber filters and frozen until analysis. Algal toxins were measured using Microcystin ELISA method with a minimum reporting level of $0.10 \mu \mathrm{g} / \mathrm{L}$.

\section{Integrated Water Samples}

POCIS from the field and all blank samplers were individually extracted at CERC using $25 \mathrm{~mL}$ of methanol (Alvarez, 2010). Two of the extracts from each site (and from each field blank) were concentrated using rotary evaporation and nitrogen blowdown, sealed in 1-mL amber glass ampules, and shipped to the NWQL for analysis. The POCIS extracts were analyzed for concentrations of current-use pesticides (appendix B, table B3) and pharmaceuticals (appendix B, table B4).

POCIS pesticide samples were prepared at the NWQL by transferring $90 \mu \mathrm{L}$ of extract to an analytical vial, diluted with water $(900 \mu \mathrm{L})$ and a mixture of internal standards $(10 \mu \mathrm{L})$. Dilution of POCIS extracts was required because most extracts contained co-extracted matrix material that causes suppression of internal standards. Laboratory blank and laboratory-fortified spike samples were prepared using comparable volumes of water and internal standard and processed with the POCIS extracts (Alvarez, 2010).

At the NWQL, POCIS extracts for pharmaceutical analysis were solvent exchanged from methanol to water. For environmental POCIS extracts, an aliquot of the solvent-exchanged extract was diluted 100:1 with water to minimize saturating detector response; field and fabrication blanks were analyzed undiluted. Prior to analysis, $995 \mu \mathrm{L}$ of the solvent exchanged, diluted (environmental POCIS samples) or undiluted (field and fabrication blank samples), were transferred to an analytical vial and a mixture of the internal standards $(5 \mu \mathrm{L})$ was added. 
Daily-composite samples (vials 1 through 7) from the pesticide autosamplers were analyzed by the EPA Office of Pesticide Program (OPP). Sample splits of the weekly composite sample (vial 9) and the spike sample (vial 8) were analyzed for pesticide concentrations by the NWQL and the OPP laboratory. Analytical Service Request (ASR) forms (USGS) and cooler inventory forms (USGS and EPA) were included with sample shipments. Bar codes were affixed to each vial as an auxiliary data match and tracking method. Weekly composite and spike samples from the pesticide autosamplers were analyzed for pesticides (appendix B, table B3) following the same method as the discrete water samples (Sandstrom and others, 2015).

\section{Discrete Sediment Samples}

Weekly discrete whole-water samples were analyzed for suspended-sediment concentrations at the USGS Cascade Volcano Observatory Sediment Laboratory in Vancouver, Washington. Methods for processing suspended-sediment concentrations are described in Guy (1969) and Knott and others (1993) and used a wet-sieving filtration method.

Streambed samples collected during the ecological sampling were homogenized using a power mixer and then divided into aliquots for analyses of various chemical constituents and for bioassays (toxicity testing). Sediment aliquots were analyzed for organic wastewater indicator compounds by solid-phase extraction and capillary column gas chromatography/mass spectrometry (CC GC/MS) (appendix B, table B6; Burkhardt and others, 2006) and for PAHs and other semi-volatile compounds by solid-phase extraction and GC/MS (appendix B, table B7; Zaugg and others, 2006b). For organochlorine insecticides, PCBs, and PBDEs in sediment, a custom method was used that extracted the sample by pressurized liquid extraction and solid-phase extraction and analyzed the compounds by electron-capture negative ionization mode (GC/ECNIMS) with ammonia reagent gas (appendix B, table B8; Mahler and others, 2009; Wagner and others, 2014). Streambed sediment was analyzed for 118 current-use pesticides at the USGS Pesticide Fate Research Laboratory (Sacramento, California) by gas chromatography-tandem mass spectrometry (Hladik and McWayne, 2012).

Hormone compounds in sediment were analyzed by gas chromatography-tandem mass spectrometry (appendix B, table B9; Foreman and others, 2012). Major and trace elements in sediment were analyzed by the USGS Crustal Geophysics and Geochemistry Laboratory (Denver, Colorado) using inductively coupled plasma-mass spectrometry (ICP-MS) following dissolution in a mixture of hydrochloric, nitric, perchloric, and hydrofluoric acids (Smith and others, 2013). An aliquot of streambed sediment was sieved to less than $63 \mu \mathrm{m}$ and analyzed for major and trace elements (appendix $\mathrm{B}$, table B10) by ICP-MS (Smith and others, 2013) at the Crustal laboratory and for radionuclides $\left({ }^{7} \mathrm{Be}\right.$,

${ }^{137} \mathrm{Cs},{ }^{226} \mathrm{Ra}$, and ${ }^{210} \mathrm{~Pb}$ ) at the USGS Sediment Radioisotope Laboratory in Menlo Park, California. The radionuclides were analyzed using a high-resolution gamma spectrometer with an intrinsic germanium detector following methods described in Van Metre and others (2004).

\section{Integrated Sediment Samples}

Suspended sediment samples collected from the Walling tubes were concentrated in the laboratory prior to shipment. The 5-gal buckets containing water and sediment were allowed to settle at $4{ }^{\circ} \mathrm{C}$ for one week or longer, depending on clarity of the overlying water. After settling, overlying water was poured off to reduce the sediment-water slurry for transfer to a 1- to 3-L plastic sample bottle. Sample bottles were then capped, sealed with tape, and shipped overnight on ice to the USGS Maryland Water Science Center. Suspended sediment samples from the Walling tubes were analyzed for major and trace elements by ICP-MS at the USGS Crustal Geophysics and Geochemistry Laboratory and for radionuclides at the USGS Sediment Radioisotope Laboratory. 


\section{Ecological Samples}

Samples for chlorophyll $a$, pheophytin $a$, and periphyton ash-free dry mass were collected during the ecological survey and processed in the field by filtering onto $0.47-\mu \mathrm{m}$ glass-fiber filters. The filters were analyzed using standard methods and EPA method 445.0, respectively, by the NWQL (appendix B, table B11; Arar and Collins, 1997; American Public Health Association, 1998). Benthic macroinvertebrate samples were processed by the Biological Unit of the NWQL using the quantitative fixed count method (Moulton and others, 2000). Briefly, the sample is sorted to attain a minimum of 300 organisms to which taxonomic identifications generally are made to the species or genus level, which is similar to the method described in the US EPA Rapid Bioassessment Protocol III. Quality assurance is verified in both the sorting and taxonomic steps by a second person on 10 percent of the organisms (Moulton and others, 2000). Taxonomic and enumeration results were uploaded to BioData (U.S. Geological Survey, 2016), the Water Mission Area database for aquatic bioassessment data.

Benthic algae samples were analyzed for diatom community composition and abundance at the Institute of Arctic and Alpine Research (INSTARR) Laboratory (University of Colorado, Boulder) following NAWQA protocols (Charles and others, 2002) with the following modification. Four replicate slides were made using Battarbee chambers to obtain random distribution of cells on cover slips (Battarbee, 1973). A pre-count voucher flora was created based on examination of 80 percent of the algal slides. The voucher flora included images of all taxa encountered, with a greater number of images for rare and unknown taxa. The images were sorted into operational taxonomic units (OTUs) and assigned OTU codes. Samples and their order of analysis were randomly assigned to two analysts. Ten percent of samples were re-analyzed by each analyst and 10 percent of samples were analyzed in cross comparison. Finally, OTU codes were translated to formal scientific names following the taxonomy in the USGS BioData program and Diatoms of the United States (Spaulding and others, 2010). Voucher slides, digested material, and the voucher flora were archived at INSTAAR.

\section{Sediment Toxicity Testing}

A detailed description of the toxicity test conditions is provided in Moran and others (2017) and briefly summarized here. The toxicity tests were conducted following methods outlined in U.S. Environmental Protection Agency (2000) and in American Society for Testing and Materials International (2014a) for sediment testing, and in American Society for Testing and Materials International (2014b) for mussel testing. Whole sediment toxicity tests were conducted with the amphipod Hyalella azteca (28-day exposures), with the midge Chironomus dilutus (10-day exposures), and with the mussel Lampsilis siliquoidea (28-day exposures). As much as $1.8 \mathrm{~L}$ (assuming a 50/50 split of solids and liquids) of the composited streambed sediment were used for toxicity testing. Each species tested included endpoints of survival, weight, and biomass of test organisms. Exposures were conducted at $23{ }^{\circ} \mathrm{C}$ in $300-\mathrm{mL}$ beakers containing 10 test organisms fed daily and $100 \mathrm{~mL}$ of sediment with 2 volume additions per day of overlying water. 


\section{Quality Assurance and Quality Control}

Quality assurance $(\mathrm{QA})$ and quality control $(\mathrm{QC})$ procedures maintain the integrity, accuracy, and legal defensibility of results from data collection and assessment. Documented USGS QA/QC policies and procedures were implemented in the PNSQA study to ensure that the data can be interpreted properly and be scientifically defensible (Mueller and others, 1997; U.S. Geological Survey, 2006). QC samples were collected to identify, quantify, and document bias and variability in data that result from the sampling procedure (through field QC sampling) and laboratory procedures (through laboratory QC sampling). Field QC sampling captures bias and variability from sample collection, processing, shipping, and handling of samples. Laboratory QC sampling documents the variability of analytical methods and sample preparation in the laboratory. To ensure that all field crews followed consistent sample collection and processing procedures, classroom training was held for field personnel prior to the sampling period. Additionally, many personnel worked through a full suite of sample collection and sample processing procedures at one of the PNSQA sites prior to the start of the weekly sampling period.

The QC samples included field blanks, matrix spikes, and replicates (table 5; appendix A, table A3). Field blanks were used to test if cleaning procedures would adequately remove any sampling equipment contamination introduced by samples obtained at previous sites and ensure that sample collection, processing, handling, and shipping did not result in contamination (Mueller and others, 1997; U.S. Geological Survey, 2006). Field replicates were used to test the precision of analyses at the laboratory and were prepared by dividing a single volume of water into two samples in the field. When these samples were collected from the churn, either filtered or not, two containers were filled sequentially. When grab samples were collected, replicates were collected sequentially directly from the stream. These replicates provided a measure of the variability introduced during sample processing and analysis (Mueller and others, 1997; U.S. Geological Survey, 2006). Field and laboratory matrix spikes were used to assess the potential bias for analytes in a particular sample matrix. Bias is estimated from spiked samples by calculating the percentage of the added analyte (spike material) measured (recovered) in the sample at the laboratory (Mueller and others, 1997; U.S. Geological Survey, 2006). Recovery can be either greater than or less than 100 percent, so the bias can be either positive or negative; however, matrix interference and analyte degradation generally result in a negative bias.

Field blanks were collected once from 26 to 32 sites for each of the basic laboratory schedules (major ions, nutrients, dissolved organic carbon, pesticides, and glyphosate by immunoassay) sampled weekly (table 5). For QA/QC samples collected as part of NAWQA, Mueller and others (1997) recommend 1 field blank or replicate per every 30 (3.3 percent) or 20 (5 percent) environmental samples for the previously mentioned constituents when sampling at long-term sites; however, if a large number of environmental samples are collected in a short period of time, as was the case in the PNSQA study, it is recommended to lower the QC sample frequency to 1 per month. Therefore, for the PNSQA study, the recommended percentage was computed as 1 monthly QC sample at 70 sites or 1.4 percent. Actual field blanks represented 3.4-4.1 percent of the environmental samples, and split replicates for the same analyses represented 4.1 percent of the environmental samples, which met the recommended frequency (table 5; Mueller and others, 1997). 
Table 5. Summary counts of environmental, field blank, replicate, and spike samples of stream water from the 88 stream sites sampled in the Pacific Northwest Stream Quality Assessment study of the U.S. Geological Survey National Water-Quality Assessment Project, 2015.

[Recommended percentage from Mueller and others (1997); QA, quality assurance; KS OGRL, U.S. Geological Survey Kansas Organic Geochemistry Research Laboratory; QC, quality control; --, no data]

\begin{tabular}{|c|c|c|c|c|}
\hline Laboratory schedule & Type of sample & $\begin{array}{l}\text { Sample } \\
\text { counts }\end{array}$ & \multicolumn{2}{|c|}{$\begin{array}{l}\text { Ratio of QA to } \\
\text { environmental samples } \\
\text { (percentage) }\end{array}$} \\
\hline \multirow[t]{4}{*}{ Major ions } & Environmental & 772 & Actual & Recommended \\
\hline & Blank & 26 & 3.4 & ${ }^{1} 1.6$ \\
\hline & Replicate & 32 & 4.1 & ${ }^{1} 1.6$ \\
\hline & Spike & 0 & 0 & 0 \\
\hline \multirow[t]{4}{*}{ Nutrients } & Environmental & 772 & Actual & Recommended \\
\hline & Blank & 26 & 3.4 & ${ }^{1} 1.6$ \\
\hline & Replicate & 32 & 4.1 & ${ }^{1} 1.6$ \\
\hline & Spike & 0 & 0 & 0 \\
\hline \multirow[t]{4}{*}{ Dissolved organic carbon } & Environmental & 772 & Actual & Recommended \\
\hline & Blank & 32 & 4.1 & ${ }^{1} 1.4$ \\
\hline & Replicate & 32 & 4.1 & ${ }^{1} 1.4$ \\
\hline & Spike & 0 & 0 & 0 \\
\hline \multirow[t]{4}{*}{ Pesticides } & Environmental & 772 & Actual & Recommended \\
\hline & Blank & 32 & 4.1 & ${ }^{1} 1.4$ \\
\hline & Replicate & 32 & 4.1 & ${ }^{1} 1.4$ \\
\hline & Spike & 70 & 9.1 & ${ }^{2} 9.1$ \\
\hline \multirow[t]{4}{*}{ Glyphosate (immunoassay) } & Environmental & 772 & Actual & Recommended \\
\hline & Blank & 32 & 4.1 & -- \\
\hline & Replicate & 32 & 4.1 & -- \\
\hline & Spike & 0 & 0 & -- \\
\hline \multirow{4}{*}{ Pharmaceuticals } & Environmental & 210 & Actual & Recommended \\
\hline & Blank & 10 & 4.8 & -- \\
\hline & Replicate & 11 & 5.2 & -- \\
\hline & Spike & 9 & 4 & -- \\
\hline \multirow[t]{4}{*}{ Organic wastewater indicators } & Environmental & 210 & Actual & Recommended \\
\hline & Blank & 10 & 4.8 & -- \\
\hline & Replicate & 11 & 5.2 & -- \\
\hline & Spike & 9 & 4.3 & -- \\
\hline \multirow[t]{4}{*}{ Glyphosate (KS OGRL) } & Environmental & 140 & Actual & Recommended \\
\hline & Blank & 6 & 4.3 & -- \\
\hline & Replicate & 7 & 5.0 & -- \\
\hline & Spike & 9 & 6.4 & -- \\
\hline
\end{tabular}




\begin{tabular}{l|c|c|cc}
\hline Laboratory schedule & Type of sample & $\begin{array}{c}\text { Sample } \\
\text { counts }\end{array}$ & $\begin{array}{c}\text { Ratio of QA to } \\
\text { environmental samples } \\
\text { (percentage) }\end{array}$ \\
\hline Mercury & Environmental & 352 & Actual & Recommended \\
& Blank & 19 & 5.4 & ${ }^{1} 1.4$ \\
& Replicate & 19 & 5.4 & ${ }^{1} 1.4$ \\
& Spike & 0 & 0 & 0 \\
\hline Isotopes & Environmental & 70 & Actual & Recommended \\
& Blank & 0 & 0.0 & -- \\
& Replicate & 14 & 20.0 & -- \\
& Spike & 0 & 0 & -- \\
\hline
\end{tabular}

${ }^{1}$ Mueller and others (1997) recommends substituting 1 per month if a large number of environmental samples are collected in a short period of time rather than a set 1 per 30 (3.3 percent) or 20 (5 percent). Therefore, for the PNSQA study, weekly samples were collected at 70 sites for 10 weeks, so the recommended percentage was computed as 1 monthly QC sample at 70 sites, or 1.4 percent.

${ }^{2}$ Recommended amount is one per site.

No recommendation for QA/QC samples was provided for the organic compounds of emerging concern (pharmaceuticals and organic wastewater indicators) in Mueller and others (1997). Field blanks and split replicates for pharmaceutical and organic wastewater indicator analyses represented 4.8 and 5.2 percent of the environmental samples, respectively (table 5). Matrix spikes were performed on all analyses for organic compounds, with the exception of glyphosate analysis by immunoassay. The frequency of these spikes ranged from 4 to 6.4 percent depending on the analyte, and was about 1 spike per every 20 environmental samples (5 percent) (table 5).

Quality assurance included maintaining standardized sample collection and handling protocols among all field personnel, as described in the National Field Manual (U.S. Geological Survey, various dates) for water and sediment sampling, and by Moulton and others (2002) for ecological sampling. All sampling and handling protocols were reviewed by field personnel involved in the PNSQA study during training courses prior to field work. Additionally, several programs exist within the USGS Branch of Quality Systems to help document the quality of project results. For laboratory analyses conducted by the NWQL, documented QC included double-blind analyses of blanks for organic and inorganic constituents, and provision of graphical and tabular control data for the analytical lines. Field personnel involved in the PNSQA study have been tested annually to verify their proficiency in collecting field data, including $\mathrm{pH}$, alkalinity, and specific conductivity.

Water-quality data from each sampling event were reviewed for completeness, precision, bias, and transcription errors when received from the laboratory as part of the QA/QC procedures. Waterquality and sediment-quality data were stored in the National Water Information System (NWIS) database. Quality-assured water-quality and sediment-quality data are available for retrieval at https://waterdata.usgs.gov/nwis and using the data retrieval application at the RSQA project Web site (https://txpub.usgs.gov/RSQA/Query.aspx). The NWQL provides all QA/QC documentation for their analytical services at https://nwql.usgs.gov/quality. 


\section{Data Management Procedures}

The final goal of the data management process for the RSQA, including PNSQA, is to have all appropriate data reviewed, approved, and stored with the appropriate data quality indicator (DQI) code in the NWIS database. Because PNSQA sampling sites were located in multiple states, data entry and retrieval for sites in a particular State were managed by the USGS Water Science Center (WSC) for that State. The NWIS database is composed of separate distributed databases that are each hosted by the WSC for the State in which the site is located. Thus, the WSC responsible for managing PNSQA data in NWIS depended on the State in which the PNSQA site was located. Additionally, a central datamanagement team was created to include both national RSQA staff and regional PNSQA staff to facilitate the data management process. Centralization of the data management process was adopted to ensure consistency among the WSCs for each RSQA and among all RSQA study areas. The nine main steps implemented for the data management process were as follows:

1. Sampling matrix and sample coding design,

2. Using electronic field forms including barcoding,

3. Checking sample status at all laboratories,

4. Checking NWIS sample records,

5. Transferring data from laboratory to NWIS,

6. Establishing project networks,

7. Checking sample coding and field parameters,

8. Checking data quality, and

9. Approving data in NWIS.

Sampling sites selected for PNSQA were assigned the appropriate network designations in NWIS ProjectNetworks to allow integration with similar sites across many regions and to designate the site type in NWIS. These network designations were obtained from the project planning documents and, where possible, were kept consistent with other network designations from previous regional studies. ProjectNetworks documentation was provided to local WSC personnel so they could establish their sites in NWIS ProjectNetworks.

Prior to the start of sampling, the PNSQA team prepared the sample matrix design and sample coding plan for all aspects of the field process. The sampling matrix distributed QC samples equally across sites, sample teams, and time periods for optimum coverage. The matrix also served as a summary diagram for the type, frequency, and location of environmental and QC samples to be collected (appendix A, table A3). The field data and field supply managers of the central data management team used a consistent sample coding scheme among the PNSQA sampling teams to ensure a well-structured and manageable dataset. Additionally, training and written guidelines for sampling coding were made available to sampling teams prior to the start of sampling.

Weekly sample bottle packs were assembled at a central location by the field supply manager and consisted of the appropriate bottles, filters, preservatives, labels, and ASRs for each stream site; bottle packs were shipped to the local WSC at least 1 week prior to sampling. Centralizing the creation of sample bottle packs helped ensure that correct sample coding, sample schedules, and timing of QC samples matched the proposed sample plan and reduced errors in the sample login process at the analytical laboratories. 
The PNSQA sampling teams from all the WSCs used the Personal Computer Field Form (PCFF) version 7.2 software created by the USGS, which provides electronic field forms for data collection at sampling sites. The PCFF software streamlines the process of uploading (logging in) field data and sampling codes to NWIS by automatically generating the batch load files required by NWIS (qwsample and qwresult), thereby resulting in a more efficient process of data flow from field and laboratory to database. The information uploaded to NWIS for each sample is stored under a unique number associated with that sample, as are later results received from the laboratory.

Sample shipment schedules were established prior to the start of sampling for PNSQA, which generally took place twice per week (appendix A, table A2). Sampling teams and other WSC personnel were responsible for the shipment process. The field data manager of the central data-management team continuously tracked the shipments to verify that the shipped samples were received at all laboratories (1) within the correct holding times, (2) in the proper condition (for example, chilled samples received at the appropriate temperature of $4{ }^{\circ} \mathrm{C}$ or less), and (3) with proper documentation. The field data manager worked with the laboratories to correct problems with mislabeled samples or ASRs in a timely manner and to communicate problem-resolution approaches to WSC personnel. During this process, the field data manager also established the connection between the USGS Laboratory Information Management System (LIMS) used to transfer sample results and the NWIS database used to receive and store sample results.

During sampling and the corresponding establishment of sample records in NWIS, the field data manager of the central data-management team inspected sample coding and procedures of sample records in NWIS to ensure that samples were established properly and in a consistent manner. Sample coding or procedures were modified if found to be inaccurate or inconsistent. These modifications involved changes or corrections to sample time offsets, sample type coding, or other documentation at the laboratory or in NWIS. Modifications in sample coding or procedures related to data management or sample submittal were communicated immediately to sampling teams to ensure that appropriate adjustments were made before the next sampling.

Most laboratories used for sample analysis by PNSQA transmitted sample results through the Water Quality Data Exchange (QWDX) for automatic upload into the NWIS database using manually created batch files. Batch files were created by the field data manager and loaded into the respective WSC NWIS host. The field data manager verified that the manually loaded data were properly loaded into NWIS. Data files provided through email by laboratories and data not applicable to NWIS (for example CERC toxicity data), were stored electronically in the RSQA team database rather than NWIS. These data will be made available using the ScienceBase digital publishing site supported by the USGS.

After sampling was completed, the field data manager inspected the NWIS sample records for completeness regarding field data collection, including stream measurements (streamflow, stage, sampling points, stream width, and so forth), field parameters ( $\mathrm{pH}$, air and water temperature, specific conductance, dissolved oxygen), and sample coding (sample purpose, purpose of site visit, sampling method, sampler type, and multiple QC-related sample codes). Manual checks were made for each sample and any corrections were communicated to WSC personnel. The field data manager made any needed changes in NWIS. 
National RSQA team members reviewed the water-quality and sediment-quality results received from the laboratory. The water-quality data reviews included identification and review of extremes in the data (outliers), inconsistencies or unexpected results in the data, and major differences between environmental samples and replicates, detected values in blanks, and analyte recoveries in spike samples. These RSQA team members communicated request reruns, reloads, and verification of results from the laboratory; team members involved in the review process worked closely with the field data manager to verify the completeness of sample results, and a final dataset was established in NWIS and the RSQA team database.

Upon completion of the data review process by the RSQA team members, the field data manager provided the appropriate WSC personnel with a table of the data review results from the RSQA Team Database for internal review. WSC personnel subsequently changed the DQI codes for each individual water-quality parameter, based on the results of the review, to reviewed and accepted (R) or reviewed and rejected $(\mathrm{Q})$. Data that were considered reviewed and rejected were not used in data analysis or publications.

\section{Summary}

This report summarizes the study design and methods used during an intensive regional study to assess stream quality in the Pacific Northwest. Eighty-eight wadeable stream sites were selected throughout the Puget Lowland and Willamette Valley Level III Ecoregions to determine the occurrence and levels of multiple stressors and to assess the condition of aquatic biological communities. Water quality was determined during a 4- to 10-week index period from April to June 2015 followed by an ecological survey of in-stream physical habitat and biological communities for algae, invertebrates, and fish. Multiple parameters covering a wide variety of potential sources were measured during this study and included discrete, integrative, and continuous water-quality sampling. The overall goal of the project was to improve our understanding of multiple water-quality stressors that affect wadeable streams throughout the region by evaluating relations between these stressors and indicators of ecological health.

\section{References Cited}

Alvarez, D.A., 2010, Guidelines for the use of the semipermeable membrane device (SPMD) and the polar organic chemical integrative sampler (POCIS) in environmental monitoring studies: U.S. Geological Survey Techniques and Methods, book 1, chap. D4, 28 p.

American Public Health Association, 1998, Standard methods for the examination of water and wastewater (20th ed.): Washington, D.C., American Public Health Association, American Water Works Association, and Water Environment Federation, p. 3-37-3-43.

American Public Health Association, American Water Works Association, and Water Environment Federation, 1998, Standard methods for the examination of water and wastewater (20th ed.): Washington, D.C., American Public Health Association, American Water Works Association, and Water Environment Federation, p 3-37 to 3-43.

American Society for Testing and Materials International, 2014b, Standard guide for conducting laboratory toxicity tests with freshwater mussels: Report ASTM E2455-06 (2013), Annual Book of American Society for Testing and Materials International Standards Volume 11.06, West Conshohocken, Pennsylvania, accessed May 6, 2015, at http:/www.astm.org/Standards/E2455.htm. 
American Society for Testing and Materials International, 2014a, Standard test method for measuring the toxicity of sediment associated contaminants with freshwater invertebrates: Report ASTM E170605(2010), Annual Book of American Society for Testing and Materials International Standards Volume 11.06, West Conshohocken, Pennsylvania, accessed May 6, 2015, at http://www.astm.org/Standards/E1706.htm.

Arar, E.J., and Collins, G.B., 1997, U.S. Environmental Protection Agency method 445.0-In vitro determination of chlorophyll $a$ and pheophytin $a$ in marine and freshwater algae by fluorescence (revision 1.2): Cincinnati, Ohio, U.S. Environmental Protection Agency, National Exposure Research Laboratory, Office of Research and Development.

Battarbee, R.W. 1973. A new method for the estimation of absolute microfossil numbers, with reference especially to diatoms: Limnology and Oceanography, v. 18, no. 4, p. 647-653.

Brown, L.R., Gray, R.H., Hughes, R.H., and Meador, M.R., eds., 2005, Effects of urbanization on stream ecosystems: Bethesda, Maryland, American Fisheries Society, Symposium 47, 423 p.

Burkhardt, M.R., Zaugg, S.D., Smith, S.G., and ReVello, R.C., 2006, Determination of wastewater compounds in sediment and soil by pressurized solvent extraction, solid-phase extraction, and capillary-column gas chromatography/mass spectrometry: U.S. Geological Survey Techniques and Methods, book 5, chap. B2, 33p.

Charles, D.F., Knowles, C., and Davis, R.S., 2002, Protocols for the analysis of algal samples collected as part of the U.S. Geological Survey National Water-Quality Assessment Program: Philadelphia, The Academy of Natural Sciences, Report No. 02-06, 124 p., https://diatom.ansp.org/nawqa/Protocols.aspx.

Coles, J.F., McMahon, G., Bell, A.H., Brown, L.R., Fitzpatrick, F.A., Eikenberry-Scudder, B.C., Woodside, M.D., Cuffney, T.F., Bryant, W.L., Cappiella, K., Fraley-McNeal, L., and Stack, W.P., 2012, Effects of urban development on stream ecosystems in nine metropolitan study areas across the United States: U.S. Geological Survey Circular 1373, 138 p.

Coplen, T.B., Qi, Haiping, Révész, Kinga, Casciotti, Karen, and Hannon, J.E., 2012, Determination of the $\delta^{15} \mathrm{~N}$ and $\delta^{18} \mathrm{O}$ of nitrate in water; RSIL lab code 2900, chap. 17 of Stable isotope-ratio methods, section C, of Révész, Kinga, and Coplen, T.B. eds., Methods of the Reston Stable Isotope Laboratory (slightly revised from version 1.0 released in 2006): U.S. Geological Survey Techniques and Methods, book 10, 35 p., https://pubs.usgs.gov/tm/2006/tm10c17/.

Davis, B.E., 2005, A guide to the proper selection and use of federally approved sediment and waterquality samplers: U.S. Geological Survey Open-File Report 2005-1087, 20 p.

DellaSala, D., Orians, G., Kavanagh, M., and Sims, K., 2001, Puget lowland forest (NA0524): World Wildlife Fund, Wildfinder Terrestrial Ecoregions, accessed July 2007, at http://www.worldwildlife.org/science/wildfinder/profiles/ na0524.html.

De Wild, J.F., Olson, M.L., and Olund, S.D., 2002, Determination of methyl mercury by aqueous phase ethylation, followed by gas chromatographic separation with cold vapor atomic fluorescence detection: U.S. Geological Survey Open-File Report 01-445, 14 p., accessed October 19, 2009, at https://pubs.er.usgs.gov/usgspubs/ofr/ofr2001445.

Dunham, J., Chandler, G., Rieman, B., and Martin, D., 2005, Measuring stream temperature with digital data loggers-A user's guide: U.S. Department of Agriculture General Technical Report RMRSGTR-150WWW, Forest Service, Rocky Mountain Research Station, 16 p., accessed December 19, 2014, at https://www.fs.fed.us/rm/pubs/rmrs_gtr150.pdf.

Fishman, M.J., ed., 1993, Methods of analysis by the U.S. Geological Survey National Water Quality Laboratory-Determination of inorganic and organic constituents in water and fluvial sediments: U.S. Geological Survey Open-File Report 93-125, 217 p. 
Fishman, M.J., and Friedman, L.C., 1989, Methods for determination of inorganic substances in water and fluvial sediments: U.S. Geological Survey Techniques of Water-Resources Investigations, book 5, chap. A1, 545 p.

Fitzpatrick, F.A., White, I.R., D’Arconte, P.J., Meador, M.R., Maupin, M.A., and Gurtz, M.E., 1998, Revised methods for characterizing stream habitat in the National Water-Quality Assessment Program: U.S. Geological Survey Water-Resources Investigations Report 98-4052, 67 p.

Foreman, W.T., Gray, J.L., ReVello, R.C., Lindley, C.E., Losche, S.A., and Barber, L.B., 2012, Determination of steroid hormones and related compounds in filtered and unfiltered water by solidphase extraction, derivatization, and as chromatography with tandem mass spectrometry: U.S. Geological Survey Techniques and Methods, book 5, chap. B9, 131 p.

Furlong, E.T., Noriega, M.C., Kanagy, C.J., Kanagy, L.K., Coffey, L.J., and Burkhardt, M.R., 2014, Determination of human-use pharmaceuticals in filtered water by direct aqueous injection-Highperformance liquid chromatography/tandem mass spectrometry: U.S. Geological Survey Techniques and Methods, book 5, chap. B10, 49 p., accessed April 3, 2015, at https://dx.doi.org/10.3133/tm5B10. Furlong, E.T., Werner, S.L., Anderson, B.D., and Cahill, J.D., 2008, Determination of human-health pharmaceuticals in filtered water by chemically modified styrene-divinylbenzene resin-based solidphase extraction and high-performance liquid chromatography/mass spectrometry: U.S. Geological Survey Techniques and Methods, book 5, sec. B, chap. B5, 56 p.

Garrett, J.D., Frey, J.W., Van Metre, P.C., Journey, C. A., Nakagaki, Naomi, Button, D.T., and Howell, L.H., 2017, Design and Methods of the Midwest Stream Quality Assessment (MSQA), 2013: U.S. Geological Survey Open-File Report 2017-1073, 59 p. and 4 appendixes, https://doi.org/10.3133/ofr20171073.

Gellis, A.C., Fuller, C.C., and Van Metre, P.C., 2016, Sources and ages of fine-grained sediment to streams using fallout radionuclides in the Midwestern United States: Journal of Environmental Quality, http://dx.doi.org/10.1016/j.jenvman.2016.06.018.

Gilliom, R.J., Barbash, J.E., Crawford, C.G., Hamilton, P.A., Martin, J.D., Nakagaki, N., Nowell, L., Scott, J.C., Stackelberg, P.E., Thelin, G.P., Wolock, D.M., 2006, Pesticides in the Nation's streams and ground water, 1992-2001: U.S. Geological Survey Circular 1291, 172 p.

Glover, J.B., Eidson, J.P., Eargle, D.A., Renfrow, R.T., Castleberry, W.S., and Domino, M.E., 2008, The influence of land use on aquatic macroinvertebrates in streams and rivers of South Carolina: Proceedings of the 2008 South Carolina Water Resources Conference, Charleston, South Carolina, October 14-15, 2008.

Gosz, J.R., 1992, Gradient analysis of ecological change in time and space-Implications for forest management: Ecological Applications, v. 2, no. 3, p. 248-261.

Gregory, M.B., and Calhoun, D.L., 2006, Physical, chemical, and biological responses of streams to increasing watershed urbanization in the Piedmont ecoregion of Georgia and Alabama, 2003: U.S. Geological Survey Scientific Investigations Report 2006-5101-B, 104 p.

Guy, H.P., 1969, Laboratory theory and methods for sediment analysis: U.S. Geological Survey Techniques of Water-Resources Investigations, book 5, chap. C1, 58 p.

Hambrook, J.A., and Canova, M.G., 2007, Algal biomass indicators (ver. 1.0): U.S. Geological Survey Techniques of Water-Resources Investigations, book 9, chap. A7, section 7.4, accessed December 2, 2016, at https://pubs.water.usgs.gov/twri9A6/.

Herlihy, A.T., Hughes, R.M., and Sifneos, R.C., 2006, National clusters of fish species assemblages in the conterminous United States and their relationship to existing landscape classification schemes, in Hughes, R.M., Wang, L., and Seelbach, P.W., eds., Influences of landscapes on stream habitats and biological assemblages: Bethesda, Maryland, American Fisheries Society, p. 87-112. 
Herlihy, A.T., Paulsen, S.G., Sickle, J.V., Stoddard, J.L., Hawkins, C.P., and Yuan, L.L., 2008, Striving for consistency in a national assessment-The challenges of applying a reference-condition approach at a continental scale: Journal of the North American Benthological Society, v. 27, no. 4, p. 860-877, http://dx.doi.org/10.1899/08-08101.

Hladik, M.L., and McWayne, M.M., 2012, Methods of analysis - Determination of pesticides in sediment using gas chromatography/mass spectrometry: U.S. Geological Survey Techniques and Methods, book 5, chap. C3, 18 p. [Also available at https://pubs.usgs.gov/tm/tm5c3.]

Homer, C.G., Dewitz, J.A., Yang, L., Jin, S., Danielson, P., Xian, G., Coulston, J., Herold, N.D., Wickham, J.D., and Megown, K., 2015, Completion of the 2011 National Land Cover Database for the conterminous United States-Representing a decade of land cover change information:

Photogrammetric Engineering and Remote Sensing, v. 81, no. 5, p. 345-354.

Journey, C.A., Van Metre, P.C., Bell, A.H., Garrett, J.D., Button, D.T., Nakagaki, N., Qi, S.L., and Bradley, P.M., 2015, Design and methods of the Southeast stream quality assessment (SESQA), 2014: U.S. Geological Survey Open-File Report 2015-1095, 46 p., https://dx.doi.org/10.3133/ofr20151095.

Klemm, D.J., Blocksom, K.A., Fulk, F.A., Herlihy, A.T., Hughes, R.M., Kaufmann, P.R., Peck, D.V., Stoddard, J.L., Thoeny, W.T., Griffith, M.B., and Davis, W.S., 2003, Development and evaluation of a macroinvertebrate biotic integrity index (MBII) for regionally assessing mid-Atlantic highlands streams: Environmental Management, v. 31, p. 656-669.

Knott, J.M., Glysson, G.D., Malo, B.A., and Schroeder, L.J., 1993, Quality assurance plan for the collection and processing of sediment data by the U.S. Geological Survey, Water Resources Division: U.S. Geological Survey Open-File Report 92-499, accessed November 17, 2010, at https://pubs.er.usgs.gov/pubs/ofr/ofr92499.

Lenat, D.R., and Crawford, J.K., 1994, Effects of land use on water quality and aquatic biota of three North Carolina Piedmont streams: Hydrobiologia, v. 294, no. 3, p. 185-199.

Lewis, M.E., and Brigham, M.E., 2004, Low level mercury: U.S. Geological Survey Techniques of Water-Resources Investigations, book 9, chap. A5, section 5.6.4.B, 26 p., accessed March 16, 2007, at https://water.usgs.gov/owq/FieldManual/chapter5/pdf/5.6.4.B_v1.0.pdf.

Loftin, K.A., Clark, J.M., Journey, C.A., Kolpin, D.W., Van Metre, P.C., Carlisle, D. and Bradley, P.M., 2016, Spatial and temporal variation in microcystin occurrence in wadeable streams in the southeastern United States: Environmental Toxicology and Chemistry, v. 35, p. 2,281-2,287, doi:10.1002/etc.3391.

Mahler, B.J., VanMetre, P.C., Burley, T.E., Loftin, K.A., Meter, M.T., and Nowell, L.H., 2017, Similarities and differences in occurrence and temporal fluctuations in glyphosate and atrazine in small Midwestern streams (USA) during the 2013 growing season: Science of the Total Environment, v. 579, p. 149-158, http://dx.doi.org/10.1016/j.scitotenv.2016.10.236.

Mahler, B.J., VanMetre, P.C., Wilson, J.T., and Musgrove, M, 2009, Fipronil and its degradates in indoor and outdoor dust: Environmental Science and Technology, v. 43, p. 5,665-5,670.

McMahon, G., Gregonis, S.M., Waltman, S.W., Omernik, J.M., Thorson, T.D., Freeouf, J.A., Rorick, A.H., and Keys, J.E., 2001, Developing a spatial framework of common ecological regions for the conterminous United States: Environmental Management, v. 28, no. 3, p. 293-316.

Meyer, M.T., Loftin, K.A., Lee, E.A., Hinshaw, G.H., Dietze, J.E., and Scribner, E.A., 2009, Determination of glyphosate, its degradation product aminomethylphosphonic acid, and glufosinate, in water by isotope dilution and online solid-phase extraction and liquid chromatography/tandem mass spectrometry: U.S. Geological Survey Techniques and Methods book 5, chap. A10, 32 p. [Also available at https://pubs.usgs.gov/tm/tm5a10/pdf/tm5a10.pdf.] 
Moran, P.W., Nowell, L.H., Kemble, N.E., Mahler, B.J., Waite, I.R., and VanMetre, P.C., 2017, Influence of sediment chemistry and sediment toxicity on macroinvertebrate communities across 99 wadable streams of the Midwestern USA: Science of the Total Environment, v. 599-600, p. 14691478.

Moulton, S.R., II, Carter, J.L., Grotheer, S.A., Cuffney, T.F., and Short, T.M., 2000, Methods of analysis by the U.S. Geological Survey National Water Quality Laboratory-Processing, taxonomy, and quality control of benthic macroinvertebrate samples: U.S. Geological Survey, Open-File Report 2000-212, 49 p.

Moulton, S.R., II, Kennen, J.G., Goldstein, R.M., and Hambrook, J.A., 2002, Revised protocols for sampling algal, invertebrate, and fish communities as part of the National Water-Quality Assessment Program: U.S. Geological Survey Open-File Report 02-150, 75 p., accessed April 3, 2015, at https://pubs.usgs.gov/of/2002/ofr-02-150/.

Mueller, D.K., Martin, J.D., and Lopes, T.J., 1997, Quality control design for surface-water sampling in the National Water-Quality Assessment Program: U.S. Geological Survey Open-File Report 97-223, $17 \mathrm{p}$.

Nagy, R.C., Lockaby, B.G., Helms, B., Kalin, L., and Stoeckel, D., 2011, Water resources and land use and cover in a humid region-The southeastern United States: Journal of Environmental Quality, v. 40, p. 867-878, accessed April 3, 2015, at http://dx.doi.org/10.2134/jeq2010.0365.

National Climatic Data Center, 2010, Climatography of the United States no. 20-1981-2010 Normals: National Climatic Data Center database, accessed November 18, 2014, at https://www.ncdc.noaa.gov/data-access/land-based-station-data/land-based-datasets/climatenormals/1981-2010-normals-data.

National Oceanic and Atmospheric Administration, 2012, Biological opinion-National Marine Fisheries Service Endangered Species Act Section 7 consultation on Environmental Protection Agency's registration of thiobencarb: National Oceanic and Atmospheric Administration, 404 p., accessed March 7, 2016, at https://www.nmfs.noaa.gov/pr/consultation/opinions/biop_thiobencarb.pdf.

National Oceanic and Atmospheric Administration, 2016, West Coast salmon and steelhead listings: National Oceanic and Atmospheric Administration Web site, accessed March 9, 2017, at http://www.westcoast.fisheries.noaa.gov/protected_species/salmon_steelhead/salmon_and_steelhead listings/salmon_and_steelhead_listings.html.

National Research Council, 2009, Urban stormwater management in the United States: Washington, D.C., National Academies Press.

O'Dell, J.W., 1993, EPA method 365.1-Determination of phosphorus by semi-automated colorimetry (rev. 2.0): Cincinnati, Ohio, U.S. Environmental Protection Agency, Office of Research and Development.

Omernik, J.M., 1987, Ecoregions of the conterminous United States [Map, scale 1:7,500,000]: Annals of the Association of American Geographers, v. 77, no. 1, p. 118-125.

Omernik, J.M., 1995, Ecoregions-A spatial framework for environmental management, in Davis, W.S., and T.P. Simon, eds., Biological assessment and criteria-Tools for water resource planning and decision making: Boca Raton, Florida, Lewis Publishers, p. 49-62.

Onset Computer Corporation, 2012, HOBO $^{\circledR}$ Water Temp Pro v2 (U22-001) manual: Onset Computer Corporation user manual 10366-G, accessed December 13, 2013, at http://www.onsetcomp.com/files/manual_pdfs/10366-G-MAN-U22-001.pdf.

Onset Computer Corporation, 2014, HOBO® U20 Water Level Logger (U20-001-0x and U20-0010x-Ti) manual: Onset Computer Corporation user manual 12315-F, accessed December 31, 2014, at http://www.onsetcomp.com/files/manual_pdfs/12315-F-MAN-U20.pdf. 
Oregon Department of Fish and Wildlife, 2006, The Oregon Conservation Strategy-Willamette Valley Ecoregion: Oregon Department of Fish and Wildlife, p. 234-235. [Also available at https://www.dfw.state.or.us/conservationstrat-egy/read_the_strategy.asp.]

Patton, C.J., and Kryskalla. J.R., 2003, Methods of analysis by the U.S. Geological Survey National Water Quality Laboratory_Evaluation of alkaline persulfate digestion as an alternative to Kjeldahl digestion for determination of total and dissolved nitrogen and phosphorus in water: U.S. Geological Survey Water-Resources Investigations Report 03-4174, 33p.

Patton, C.J., and Kryskalla, J.R., 2011, Colorimetric determination of nitrate plus nitrite in water by enzymatic reduction, automated discrete analyzer methods: U.S. Geological Survey Techniques and Methods, book 5, chap. B8, 34 p.

Phillips, J.M., Russell, M.A., and Walling, D.E., 2000, Time-integrated sampling of fluvial suspended sediment-A simple methodology for small catchments: Hydrological Processes, v. 14, p. 2,5892,602 .

Puget Sound Regional Council, 2016, Puget Sound trends-Population change and net migration: Puget Sound Regional Council. [Also available at http://www.psrc.org/assets/14735/Trend-Population201607.pdf.]

Radtke, D.B., 2005, Bottom-material samples: U.S. Geological Survey Techniques of Water-Resources Investigations, book 9, chap. A8, accessed February 22, 2007, at https://pubs.water.usgs.gov/twri9A8/.

Sandstrom, M.W., Kanagy, L.K., Anderson, C.A., Kanagy, C.J., 2015, Determination of pesticides and pesticide degradates in filtered water by direct aqueous-injection liquid chromatography-tandem mass spectrometry: U.S. Geological Survey Techniques and Methods, book 5, chap B11, 54p.

https://dx.doi.org/10.3133/tm5B11.

Sandstrom, M.W., and Wilde, F.D., 2014, Syringe-filter procedure for processing samples for analysis of organic compounds by DAI: U.S. Geological Survey Techniques of Water-Resources Investigations, book 9, chap. A5, section 2.2B, accessed February 6, 2015, at https://water.usgs.gov/owq/FieldManual/chapter5/pdf/5.2.2.B.pdf.

Sauer, V.B., and Turnipseed, D.P., 2010, Stage measurement at gaging stations: U.S. Geological Survey Techniques and Methods, book 3, chap. A7, 45 p.

Shelton, L.R., and Capel, P.D., 1994, Guidelines for collecting and processing samples of stream bed sediment for analysis of trace elements and organic contaminants for the National Water-Quality Assessment Program: U.S. Geological Survey Open-File Report 94-458, 20 p.

Smith, D.B., Cannon, W.F., Woodruff, L.G., Solano, Federico, Kilburn, J.E., and Fey, D.L., 2013, Geochemical and mineralogical data for soils of the conterminous United States: U.S. Geological Survey Data Series 801, 19 p., https://pubs.usgs.gov/ds/801/.

Sorenson, D.G., 2012, Puget lowland ecoregion, chap. 2 of Sleeter, B.M., Wilson, T.S., and Acevedo, W., eds., 2012, Status and trends of land change in the Western United States-1973 to 2000: U.S. Geological Survey Professional Paper 1794-A, p. 43-50. [Also available at https://pubs.usgs.gov/pp/1794/a/.]

Spaulding, S.A., Lubinski, D.J. and Potapova, M., 2010, Diatoms of the United States: Boulder, University of Colorado Web site, http://westerndiatoms.colorado.edu.

U.S. Census Bureau, 2010, U.S. Census, 2010: U.S Census Web site, accessed January 2017, at https://www.census.gov/prod/www/abs/decennial/ index.htm.

U.S. Environmental Protection Agency, 1996, Method 1669-Sampling ambient water for trace metals at EPA water quality criteria levels: U.S. Environmental Protection Agency Office of Water, Engineering and Analysis Division, 37 p., accessed March 12, 2011, at https://www.epa.gov/waterscience/methods/method/inorganics/1669.pdf. 
U.S. Environmental Protection Agency, 1999, Primary distinguishing characteristics of level III ecoregions of the continental United States: U.S. Environmental Protection Agency database, accessed July 2007, at https://www.epa. gov/wed/pages/ecoregions/level_iii.htm.

U.S. Environmental Protection Agency, 2000, Methods for measuring the toxicity and bioaccumulation of sediment-associated contaminants with freshwater invertebrates ( $2 \mathrm{~d}$ ed.): U.S. Environmental Protection Agency, Office of Water Report EPA 600/R-99/064, https://water.epa.gov/polwaste/sediments/cs/upload/freshmanual.pdf.

U.S. Environmental Protection Agency, 2002, Method 1631, Revision E-Mercury in water by oxidation, purge and trap, and cold vapor atomic fluorescence spectrometry: U.S. Environmental Protection Agency, Office of Water Report EPA-821-R-02-019, 38 p.

U.S. Environmental Protection Agency, 2006, Wadeable streams assessment - A collaborative survey of the Nation's streams: U.S. Environmental Protection Agency, Office of Research and Development and Office of Water Report EPA 841-B-06-002, 82 p. plus appendix.

U.S. Environmental Protection Agency and the U.S. Geological Survey, 2012, National Hydrography Dataset Plus-NHDPlus, Version 2, Edition 2.10, geospatial data: Horizon Systems Corporation Web site, accessed January 21, 2015, at https://www.horizon-systems.com/NHDPlus/index.php.

U.S. Geological Survey, 2006, Collection of water samples (ver. 2.0): U.S. Geological Survey Techniques of Water-Resources Investigations, book 9, chap. A4, accessed October 24, 2014, at https://pubs.water.usgs.gov/twri9A4/.

U.S. Geological Survey, 2014, NLCD 2011 land cover (2011 ed., amended October 10, 2014): National Geospatial Data Asset (NGDA) Land Use Land Cover, accessed February 2015, at https://www.mrlc.gov.

U.S. Geological Survey, 2016, BioData-Aquatic bioassessment data for the Nation: U.S. Geological Survey database, https://dx.doi.org/doi:10.5066/F77W698B.

U.S. Geological Survey [various dates], National field manual for the collection of water-quality data: U.S. Geological Survey Techniques of Water-Resources Investigations, book 9, chaps. A1-A9, accessed October 19, 2009, at https://pubs.usgs.gov/twri/.

Van Metre, P.C., Wilson, J.T., Fuller, C.C., Callender, Edward, and Mahler, B.J., 2004. Collection, analysis, and age dating of sediment cores from 56 U.S. lakes and reservoirs sampled by the U.S. Geological Survey, 1992-2001. U.S. Geological Survey Scientific Investigations Report 2004-5184, $180 \mathrm{p}$.

Waite, I.R., Herlihy, A., Larsen, D.P., and Klemm, D.J., 2000, Comparing strengths of geographic and nongeographic classifications of stream benthic macroinvertebrates in the mid-Atlantic highlands, USA: Journal of the North American Benthological Society, v. 19, p. 429-441.

Wagner, R.J., Moran, P.W., Zaugg, S.D., Sevigny, J.M., and Pope, J.M., 2014, Contaminants of emerging concern in the lower Stillaguamish River Basin, Washington, 2008-11 (ver. 2.0, June 2016): U.S. Geological Survey Open-File Report 2014-1028, 14 p.

Waples, R.S., Nammack, M., Fitts, Cochrane, J.F., and Hutchings, J.A., 2013, A tale of two actsEndangered species listing practices in Canada and the United States: BioScience, v. 63, no. 9, p. 723734.

Washington State Department of Agriculture, 2001, A process for evaluating pesticides in Washington State surface waters for potential impacts to salmonids: Washington State Department of Agriculture Publication No. 057, Washington State Department of Ecology Publication No. 01-10-058.

Wilde, F.D., ed., 2004, Cleaning of equipment for water sampling (ver. 2.0): U.S. Geological Survey Techniques of Water-Resources Investigations, book 9, chap. A3, accessed December 3, 2014, at https://pubs.water.usgs.gov/twri9A3/. 
Wilde, F.D., ed. [various dates], Field measurements: U.S. Geological Survey Techniques of WaterResources Investigations, book 9, chap. A6, sections 6.0-6.8, accessed October 24, 2014, at https://pubs.water.usgs.gov/twri9A6/.

Wilde, F.D., Radtke, D.B., Gibs, Jacob, and Iwatsubo, R.T., eds., 2004 with updates through 2009, Processing of water samples (ver. 2.2): U.S. Geological Survey Techniques of Water-Resources Investigations, book 9, chap. A5, accessed October 24, 2014, at https://pubs.water.usgs.gov/twri9A5/.

Wilson, T.S., and Sorenson, D.G., 2012, Willamette Valley ecoregion, chap. 3 of Sleeter, B.M., Wilson, T.S., and Acevedo, W., eds., 2012, Status and trends of land change in the Western United States1973 to 2000: U.S. Geological Survey Professional Paper 1794-A, p. 51-57. [Also available at https://pubs.usgs.gov/pp/1794/a/.]

Zaugg, S.D., Burkhardt, M.R., Burbank, T.L., Olsen, M.C., Iverson, J.L., and Schroeder, M.P., 2006b, Determination of semivolatile organic compounds and polycyclic aromatic hydrocarbons in solids by gas chromatography/mass spectrometry: U.S. Geological Survey Techniques and Methods, book 5, chap. B3, 44 p.

Zaugg, S.D., Smith, S.G., and Schroeder, M.P., 2006a, Methods of analysis by the U.S. Geological Survey National Water Quality Laboratory-Determination of wastewater compounds in whole water by continuous liquid-liquid extraction and capillary-column gas chromatography/mass spectrometry: U.S. Geological Survey Techniques and Methods, book 5, chap. B4, 30 p. 


\section{Appendixes}

Appendixes A and B are Microsoft Excel ${ }^{\circledR}$ files and are available for download at https://doi.org/10.3133/ofr20171103.

Appendix A. Description of the Sampling Timelines, Matrix, Collection, and Processing for Water, Sediment, and Ecological Samples

Appendix B. Description of the U.S. Geological Survey Laboratory Schedules Used for Water, Sediment, and Periphyton 
Publishing support provided by the U.S. Geological Survey

Science Publishing Network, Tacoma Publishing Service Center

For more information concerning the research in this report, contact the Director, Washington Water Science Center

U.S. Geological Survey

934 Broadway, Suite 300

Tacoma, Washington 98402

https://wa.water.usgs.gov 
